\title{
In vivo Correlation Tensor MRI reveals microscopic kurtosis in the human brain on a clinical 3T scanner
}

\author{
Lisa Novello ${ }^{1, *}$, Rafael Neto Henriques, ${ }^{2, *}$, Andrada Ianuş $^{2}$, Thorsten Feiweier $^{3}$, \\ Noam Shemesh ${ }^{2,+}$ Jorge Jovicich ${ }^{1,4,+}$

\section{Center for Mind/Brain Sciences - CIMeC, University of Trento, Rovereto, Italy \\ 2 Champalimaud Research, Champalimaud Centre for the Unknown, Lisbon, Portugal. 3 Siemens Healthcare GmbH, Erlangen, Germany \\ 4 Center for Medical Sciences - CISMeD, University of Trento, Rovereto, Italy}

\footnotetext{
*Equally contributing first authors

+ Equally contributing last authors
}

Corresponding Author at:

- Lisa Novello, Center for Mind/Brain Sciences, University of Trento, Corso Bettini, 31, 38068 Rovereto, Trento, Italy. Email address: lisa.novello@unitn.it

- Rafael Neto Henriques, Champalimaud Research, Champalimaud Center for the Unknown, Av. Brasilia 1400-038, Lisbon, Portugal. Email address: rafael.henriques@neuro.fchampalimaud.org

Keywords: double-diffusion encoding; kurtosis; non-Gaussian diffusion; human brain; magnetic resonance imaging, correlation tensor MRI, CTI

Declaration of competing interests: L. Novello: None; R.N. Henriques: None; A. Ianuş: None; T. Feiweier: Thorsten Feiweier is an employee of Siemens Healthcare GmbH, owns stocks of Siemens (Healthineers) AG and holds patents filed by Siemens. N. Shemesh: None; J. Jovicich: None.

CRediT authorship contribution statement: L. Novello: Conceptualization, Methodology, Validation, Formal analysis, Investigation, Writing - original draft, Writing - review \& editing, Visualization, Data curation. R.N. Henriques: Conceptualization, Methodology, Software, Investigation, Visualization, Writing - original draft, Writing - review \& editing. A. Ianuş: Conceptualization, Methodology, Investigation, Visualization, Writing - original draft, Writing - review \& editing, Funding acquisition. T. Feiweier: Methodology, Software, Writing - review \& editing. N. Shemesh: Conceptualization, Investigation, Writing - original draft, Writing review \& editing, Supervision, Project administration, Funding acquisition. J. Jovicich: Conceptualization, Investigation, Writing - original draft, Writing - review \& editing, Supervision, Resources, Project administration, Funding acquisition. 


\section{Highlights:}

34

35

36

37

38
- Correlation Tensor MRI (CTI) was recently proposed to resolve kurtosis sources

- We implemented CTI on a 3T scanner to study kurtosis sources in the human brain

- Isotropic, anisotropic, and microscopic kurtosis sources were successfully resolved

- Microscopic kurtosis $(\mu K)$ significantly contributes to overall kurtosis in human brain

- $\mu K$ provides a novel source of contrast in the human brain in vivo 
39

\section{Abstract}

Diffusion MRI (dMRI) has become one of the most important imaging modalities for noninvasively probing tissue microstructure. Diffusion Kurtosis MRI (DKI) quantifies the degree of non-gaussian diffusion, which in turn has been shown to increase sensitivity towards, e.g., disease and orientation mappings in neural tissue. However, the specificity of DKI is limited as different microstructural sources can contribute to the total diffusional kurtosis, including: variance in diffusion tensor magnitudes $\left(K_{\text {iso }}\right)$, variance due to intravoxel diffusion anisotropy $\left(K_{\text {aniso}}\right)$, and microscopic kurtosis $(\mu K)$ related to restricted diffusion and/or microstructural disorder. The latter in particular is typically ignored in diffusion MRI signal modeling as it is assumed to be negligible. Recently, Correlation Tensor MRI (CTI) based on Double-Diffusion-Encoding (DDE) was introduced for kurtosis source separation and revealed non negligible $\mu K$ in preclinical imaging. Here, we implemented CTI for the first time on a clinical 3T scanner and investigated the kurtosis sources in healthy subjects. A robust framework for kurtosis source separation in humans is introduced, followed by estimation of the relative importance of $\mu K$ in the healthy brain. Using this clinical CTI approach, we find that $\mu K$ significantly contributes to total diffusional kurtosis both in gray and white matter tissue but, as expected, not in the ventricles. The first $\mu K$ maps of the human brain are presented. We find that the spatial distribution of $\mu K$ provides a unique source of contrast, appearing different from isotropic and anisotropic kurtosis counterparts. We further show that ignoring $\mu K-$ as done by many contemporary methods based on multiple gaussian component approximation for kurtosis source estimation - biases the estimation of other kurtosis sources and, perhaps even worse, compromises their interpretation. Finally, a twofold acceleration of CTI is discussed in the context of potential future clinical applications. We conclude that CTI has much potential for future in vivo microstructural characterizations in healthy and pathological tissue. 


\section{Introduction}

Mapping microstructural features of the brain, in vivo and non-invasively, has been a central endeavor of the MRI community for more than two decades. Diffusion-weighted MRI (dMRI) has played a key role in such microstructural characterizations due to its ability to sensitize the MRI signal towards diffusion-driven displacements, which then "sense" tissue boundaries in the range of around $>10 \mu \mathrm{m}$ (in typical clinical settings). Water molecules are highly abundant in tissues and can traverse several micrometers in a typical MR-relevant diffusion time, making dMRI an excellent indicator of tissue microstructure. At relatively low diffusion weighting, the unidirectional Apparent Diffusion Coefficient (ADC, Stejskal and Tanner, 1965; Le Bihan et al., 1986) and later the rotationally invariant Diffusion Tensor Imaging approaches (DTI, Basser et al., 1994) utilized a Gaussian diffusion framework for quantifying diffusivities, which found numerous applications from stroke detection, to white matter orientation mapping, to characterizing progressive changes in brain tissue due to plasticity (e.g. Moseley et al., 1990; Gauvin et al., 2001; Anwander et al., 2007; Roebroeck et al., 2008; Della-Maggiore et al., 2009; Scholz et al., 2009; Blumenfeld-Katzir et al., 2011; McNab et al., 2013a; Benetti et al., 2018; Hasan et al., 2018; Jacobacci et al., 2020, Yon et al., 2020, for a review see e.g. Mukherjee, 2005; Assaf and Pasternak, 2008; Johansen-Berg, 2010).

Deviations from Gaussian displacement profiles were described quite early within the framework of q-space MR (Callaghan et al., 1991; Assaf and Cohen, 1998). As high performance magnetic gradients become more readily available in clinical MRI systems, a wider range of diffusion-weighting regimes could be probed, providing evidence for nongaussian profiles also in the human brain (Mulkern et al., 1999). Since q-space gradients required very high diffusion weighting and suffer from low signal to noise, a general methodology for characterizing diffusion weighted signals at an intermediate diffusion weighting regime was elegantly introduced by Jensen et al., 2005 and Jensen \& Helpern, 2010. The ensuing Diffusion Kurtosis Imaging (DKI) methodology is a "signal representation" approach (Novikov et al., 2018; Novikov, 2021, as opposed, e.g., to microstructural models, for instance: Jespersen et al., 2007; Fieremans et al., 2011; Zhang et al., 2012; Kaden et al., 2016; Jespersen, 2018) based on the cumulant expansion of the dMRI signal up to the second order in $b$-value (with $b=\gamma^{2} \delta^{2} G^{2}(\Delta-\delta / 3)$ for ideal rectangular diffusion-sensitizing gradients, where $\gamma$ is the gyromagnetic ratio, $\delta$ is the gradient pulse duration, $\Delta$ is separation between the two leading edges of the gradient pulses, and $G$ is the gradient pulse's magnitude). By quantifying 
the kurtosis in water displacement probability, a new source of contrast for tissue microstructure was introduced (Jensen et al., 2005; Jensen \& Helpern, 2010; Wu \& Cheung, 2010). DKI has been successfully adopted to study both the healthy and the diseased human brain, providing insights into individual anatomy at high resolution (Mohammadi et al., 2015), development (Huber et al., 2019), aging (Falangola et al., 2008; Henriques, 2018), attention deficit hyperactivity disorder (ADHD, Helpern et al., 2010), neurodegenerative disorders (Arab et al., 2018) such as Alzheimer's (Gong et al., 2013, Struyfs et al., 2015) and Parkinson's disease (Wang et al., 2011; Kamagata et al., 2013; Kamagata et al., 2014; Surova et al, 2018), for characterizing brain tumors (Raab et al., 2010; Raja et al., 2016; Falk Delgado et al., 2017; Hempel et al., 2017; Hempel et al., 2018; Lin et al., 2018), and is nowadays implemented in different platforms (e.g. Leemans et al., 2009; Tabesh et al., 2011; Tournier et al., 2019; Henriques et al., 2021a).

Since DKI can be thought of as more sensitive to tissue heterogeneity, it was recognized relatively early on that it can provide higher sensitivity than its DTI counterpart (e.g. Falangola et al., 2008; Wang et al., 2011; Hui et al., 2012; Zhuo et al., 2012; Fieremans et al., 2013; Steven et al., 2014; Lin et al., 2018). However, at the same time, DKI suffers from a lack of specificity, since many factors can contribute to heterogeneity. At the voxel level, diffusion kurtosis may arise in the presence of mesoscopic effects such as the orientation dispersion of fibers and their specific configuration (Lu et al., 2006; Henriques et al., 2015). At the microscopic level (i.e. (sub)cellular level, or more generally at the level of pores or microdomains, where a microdomain can be defined as a uniform sub-voxel segment, Szczepankiewicz et al., 2015), diffusion kurtosis may arise from different sources, including the following ones (Henriques et al., 2020):

i) variance of the eigenvalues of individual diffusion tensors representing tissue microdomains, thus in the presence of shape variance (i.e. deviations of the shape of pores or microdomains from a sphere), which is referred to as anisotropic kurtosis $\left(K_{\text {aniso }}\right)$;

ii) variance across the ensemble of all microdomains' tensor magnitudes, thus in the presence of size variance, which is referred to as isotropic kurtosis ( $K_{\text {iso }}$; the subscript "iso" here refers to the isotropic part of the tensor (its magnitude), and does not require the tensor itself to be isotropic);

iii) non-Gaussian diffusion within restricting "pores" (restricted diffusion) and/or a combination of restriction and exchange in case components are exchanging, which is 
referred to as microscopic kurtosis $(\mu K$, also previously called intra-compartmental kurtosis, $K_{\text {intra, }}$ in Henriques et al., 2020).

Understanding how each of these different sources of tissue heterogeneity affect the kurtosis signal is challenging. DKI, and more generally techniques based on Single Diffusion Encoding sequences (SDE, Shemesh et al., 2016) with moderate $b$-values do not allow to determine the relative contribution of each source without making strong assumptions about tissue properties (Fieremans et al., 2011; Ianuş et al., 2016). Such assumptions, however, may affect the specificity of the derived metrics (Jelescu et al., 2015; Lampinen et al, 2017; Henriques et al., 2019), leading to potential serious errors in the "microstructural" interpretation of the metrics (Jelescu et al., 2016; Novikov et al., 2018).

Nevertheless, the ability to characterize each diffusion kurtosis source has the potential of unraveling important microstructural information that can provide new insights into tissue properties which, in turn, may have clinical impact (Szczepankiewicz et al., 2015; Szczepankiewicz et al., 2016; Nilsson et al., 2020; Alves et al., 2021). For this reason, Multidimensional Diffusion Encoding (MDE, Eriksson et al., 2013; Lasic et al., 2014, Westin et al., 2016 Shemesh et al., 2016; Topgaard, 2017), realized by combining or concatenating multiple diffusion encodings, has been developed to produce richer diffusion weighting paradigms allowing to explore correlations between different spatial dimensions, and, ultimately, to yield more specific tissue contrasts. Among MDE preparations, Double Diffusion Encoding (DDE, Mitra, 1995; Shemesh et al., 2010a; Shemesh et al., 2016, Henriques et al., 2021b) in the long mixing time regime has been used in combination with either trapezoidal or oscillating gradients (Double Oscillating Diffusion Encoding, DODE, Ianuş et al., 2017; Shemesh, 2018). Such applications stemmed from the initial theoretical ground (Mitra, 1995; Cheng \& Cory, 1999; Özarslan and Basser, 2008; Özarslan, 2009; Lawrenz et al., 2010; Jespersen \& Buhl, 2011; Jespersen, 2012) and the following experimental observations confirming that DDE provides signal differences for collinear and orthogonal diffusion gradients in elongated samples (Cheng \& Cory, 1999; Callaghan \& Komlosh, 2002) and angular modulations of the signal in the presence pores characterized by eccentricity over the plane sampled by the diffusion preparation (Shemesh et al., 2010a; Shemesh et al., 2010b; Shemesh et al., 2011; Shemesh et al., 2012a). Since then, DDE in the long mixing time regime has been extensively adopted to provide estimations of the microscopic diffusion anisotropy $(\mu A)$, a measure related to $K_{\text {aniso }}$ by the relationship $K_{\text {aniso }}=2\left(\mu A^{2} / D^{2}\right)$, with $D$ being the mean diffusivity, $\mu A^{2} \equiv 3 / 5 \operatorname{var}\left(\lambda_{\mathrm{i}}\right)$, and $\lambda_{\mathrm{i}}$ being the eigenvalues of individual diffusion tensors 
160

161

162

163

164

165

166

167

168

169

170

171

172

173

174

175

176

177

178

179

180

181

182

183

184

185

186

187

188

189

190

191

192

representing tissue microdomains (Jespersen et al., 2013; Lasic et al., 2014; Shemesh et al., 2016; Ianuş et al., 2018; Henriques et al., 2021b). The $\mu A$ measure, and its normalized version $\mu F A$, thus report on the anisotropy of the tissue at the pore or microdomain length scale (i.e. on its eccentricity), without the confounding effect of orientation dispersion at the macroscopic (i.e. at the voxel) level. DDE-derived $\mu A$ has been investigated and successfully observed in preclinical systems in both white and grey matter: in fixed grey matter (Komlosh et al., 2007), pig spinal cord (Komlosh et al., 2008), pig optic nerve and cortical grey matter, with phantoms mimicking their respective microstructures (Shemesh et al., 2010b; Shemesh et al., 2011; Shemesh and Cohen, 2011; Shemesh et al., 2012a), in both in vivo and ex vivo rat brain (Shemesh et al., 2012b, Ianuş et al., 2018; Kerkelä et al., 2020), in ex vivo monkey brain (Jespersen et al., 2013), in rat spinal cord injury (Budde et al., 2017), and in rat spinal cord ex vivo (Shemesh, 2018). In clinical systems DDE-derived $\mu A$ has been reported in pig spinal cord (Lawrenz and Finsterbusch, 2011), and successfully mapped in the in vivo healthy human brain white matter (Lawrenz and Finsterbusch, 2013; Lawrenz and Finsterbusch, 2015), cortical grey matter (Lawrenz and Finsterbusch, 2019), in the study of aging (Lawrenz et al., 2016), and in clinical applications, where it showed increased specificity compared to DTI-derived FA in multiple sclerosis lesions (Yang et al., 2018) and has been investigated in Parkinson's disease (Kamiya et al., 2020).

In addition to DDE preparations, alternative MDE pulse sequences have been adopted in parallel with the aim of resolving the kurtosis sources: previous studies showed that, under the strict Multiple Gaussian Component assumption (MGC, Henriques et al., 2020; Henriques et al., 2021c), tensor-valued information of MDE sequences can decompose $K_{\mathrm{t}}$ as the sum of $K_{\text {aniso }}$ and $K_{\text {iso }}$ (Szczepankiewicz et al., 2015, Szczepankiewicz et al., 2019a, Topgaard, 2019). While successful applications of such techniques demonstrated great promise for clinical usefulness (Szczepankiewicz et al., 2015; Szczepankiewicz et al., 2016; Andersen et al., 2020) and implementation (Szczepankiewicz et al., 2019a; Nilsson et al., 2020), a drawback of these so-called "tensor-valued" approaches is that they assume that all underlying diffusion propagators within the voxel can be approximated as multiple Gaussian components (Henriques et al., 2020; Henriques et al., 2021c). However, the validity of these assumptions has recently been called into question (Jespersen et al., 2019; Henriques et al., 2020; Henriques et al., 2021c), mainly due to the following two reasons:

i) observations of time dependent diffusion and kurtosis, a hallmark of diffusion nonGaussianity in at least one tissue compartment (Novikov et al., 2014; Fieremans et al., 
2016; Novikov et al., 2019; Lee et al., 2020a), both in preclinical systems (in vivo: Does et al., 2003; Kunz et al., 2013; ex vivo: Assaf and Cohen, 1998; Aggarwal et al., 2012; Portnoy et al., 2013; Ianuş et al., 2018; Jespersen et al., 2018) and in clinical settings in vivo (Horsfield et al., 1994; Baron and Beaulieu, 2014; Van et al., 2014; Fieremans et al., 2016; Lee et al., 2018; Grussu et al., 2019; Arbabi et al., 2020; Lee et al., 2020a; Lee et al., 2020b), with anisotropic tissues generally presenting residual orientational timedependency in isotropic encodings (Jespersen et al., 2019);

ii) the mapping of non-vanishing positive $\mu K$ values in phantoms and both in vivo and ex vivo mice at different field strengths (Paulsen et al., 2015; Henriques et al., 2020; Henriques et al., 2021c; Alves et al., 2021).

To address these concerns and resolve kurtosis sources without explicitly or implicitly relying on the MGC assumption, a new DDE strategy has been recently proposed and evaluated in preclinical systems, namely the Correlation Tensor Imaging, or CTI (Henriques et al., 2020, Henriques et al., 2021c). In particular, CTI enables the simultaneous estimation of $K_{\text {iso }}, K_{\text {aniso, }}$, and $\mu K$, by relying on the acquisition of four different DDE sets (i.e. combinations of gradient amplitude and direction) and on the cumulant expansion of DDE signals. So far, the CTI methodology has been tested in both in vivo and ex vivo rodent brains (Henriques et al., 2020, Henriques et al., 2021c; Alves et al., 2021). These studies revealed the expected contrasts for $K_{\text {aniso, }}$, the dominant kurtosis source in white matter, and for $K_{\text {iso, }}$, which highlights areas with large dispersion of microdomains' mean diffusivities such as regions with partial volume effects. Crucially, the in vivo CTI data demonstrated for the first time evidence of a positive non-vanishing $\mu K$ both in white and grey matter, with $\mu K$ being the dominant kurtosis source in grey matter (Henriques et al., 2021c). However, the CTI framework has not yet been investigated in the human brain. Together with the observation for biases in $K_{\text {aniso }}$ and $K_{\text {iso }}$ when not accounting for $\mu K$ in mice (Henriques et al., 2021c), this body of evidence prompts the translation of the CTI methodology in the clinical setting, building also on previous successful applications of DDE sequences in the study of the living human brain.

In this work, we set out to develop CTI for humans using a clinical 3T system and investigate the kurtosis sources emerging from a cohort of healthy subjects. In particular, the goals of this study are i) to evaluate the feasibility of acquiring DDE data adhering to the CTI methodology proposed in Henriques et al., 2021c, in a clinical 3T system; ii) to estimate the kurtosis sources in brain tissue derived by the CTI framework from healthy adult volunteers, 
bioRxiv preprint doi: https://doi.org/10.1101/2021.11.02.466950; this version posted November 4, 2021. The copyright holder for this preprint (which was not certified by peer review) is the author/funder, who has granted bioRxiv a license to display the preprint in perpetuity. It is made available under aCC-BY-NC-ND 4.0 International license.

225 particularly with respect to evaluating evidence for the $\mu K$ component in humans; and iii) to 226 investigate the possible implications of ignoring the $\mu K$ component, as currently established in 227 alternative approaches aiming to resolve kurtosis sources. 
A)

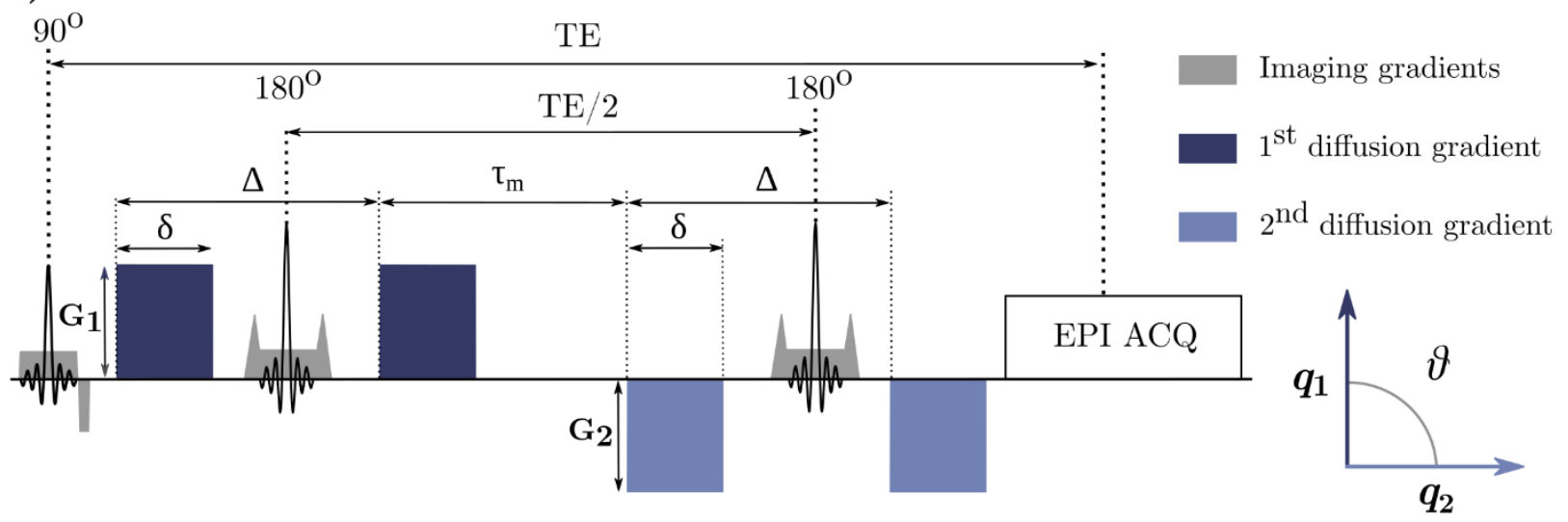

B)

Set $\begin{array}{ccc}b_{1} & b_{2} & \vartheta \\ \left(\mathrm{s} / \mathrm{mm}^{2}\right) & \left(\mathrm{s} / \mathrm{mm}^{2}\right) & (\text { degrees })\end{array}$

set $\# 1 \quad 1000 \quad 0 \quad 0$

Gradient schematic

C)

Kurtosis sources
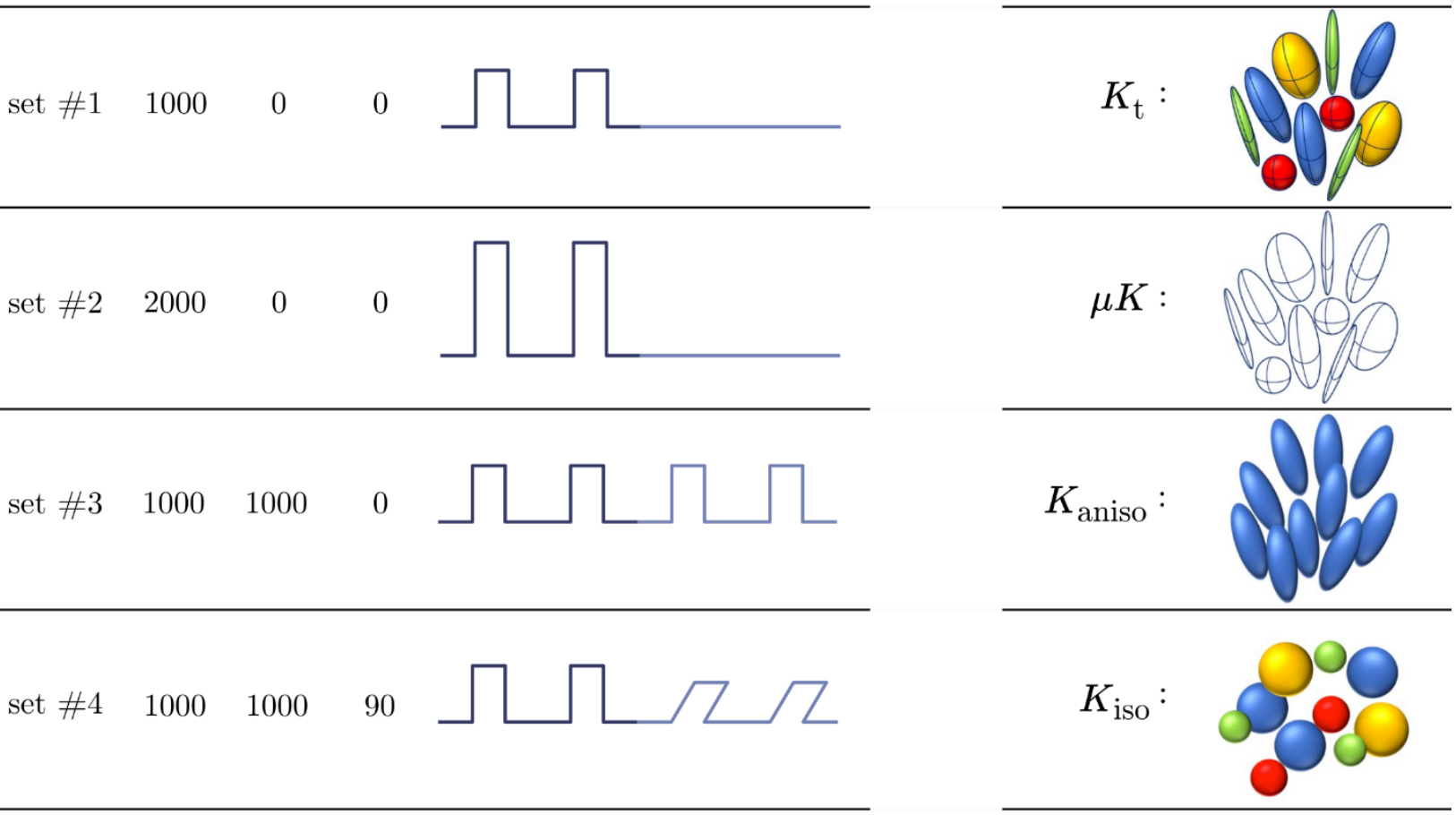

Study Protocol, $N=2$ repetitions:

Polarity of diffusion gradients:

$\begin{array}{cc}\text { repetition } \# 1 & \text { repetition } \# 2 \\ \text { "positive" } & \text { "negative" }\end{array}$

Fig. 1, CTI methodology. A) DDE sequence adopted in this study. Abbreviations: TE: Echo Time; EPI ACQ: Echo Planar Imaging acquisition module. B) CTI acquisition scheme. DDE set \#1 and DDE set \#2 mimic conventional SDE sequences. The additional DDE set \#3 and DDE set \#4 are respectively parallel and perpendicular DDE preparations, and they allow to disentangle the total Kurtosis $\left(K_{\mathrm{t}}\right)$ in its Kurtosis sources. C) Kurtosis sources. $K_{\mathrm{t}}$ can be decomposed in: i) the microscopic kurtosis $(\mu K)$, arising from non-Gaussianity induced by restricted time-dependent diffusion, structural disorder such as variations in compartmental cross-sectional area, or exchange effects, which can also be directly accessed through the combination of DDE set \#2 and DDE set \#3 (see eq. 3); ii) the anisotropic Kurtosis 
( $K_{\text {aniso }}$ ), arising from the variance in the eigenvalues describing the microdomain diffusion tensors, i.e. the shape variance, which can also be directly estimated through the combination of DDE set \#3 and DDE set \#4 (see eq. 4); and iii) the isotropic Kurtosis ( $K_{\text {iso }}$ ), arising from the variance of the microdomain diffusion tensors' mean diffusivity; factoring out exchange, $K_{\mathrm{t}}=K_{\text {aniso }}+K_{\text {iso }}+\mu K$ (Henriques et al., 2020; Henriques et al., 2021c). The acquisition was repeated twice in the same session without repositioning, with opposite polarities of all the diffusion gradients, allowing to correct for any effects

246 In the long mixing time regime, the powder average of DDE signals is equivalent to (Henriques et al., 2021c):

$$
\begin{aligned}
\log \left(\overline { E } _ { D D E } \left(b_{1},\right.\right. & \left.\left.b_{2}, \theta\right)\right) \\
& =-\left(b_{1}+b_{2}\right) \bar{D}+\frac{1}{6}\left(b_{1}^{2}+b_{2}^{2}\right) \bar{D}^{2} K_{t}+\frac{1}{2} b_{1} b_{2} \cos ^{2} \theta \bar{D}^{2} K_{\text {aniso }} \\
& +\frac{1}{6} b_{1} b_{2} \bar{D}^{2}\left(2 K_{\text {iso }}-K_{\text {aniso }}\right)+O\left(b^{3}\right)
\end{aligned}
$$

251

252

253

254

255

with $b_{1}$ and $b_{2}$ being the $b$-values of the first and of the second pair of diffusion gradients, respectively, $\theta$ being the angle between the directions of the first and the second pair of diffusion gradients, $\bar{D}$ being the mean diffusivity, and $K_{\mathrm{t}}$ being the total kurtosis of the powder averaged signal. By estimating $K_{\mathrm{t}}, K_{\text {aniso}}$, and $K_{\text {iso }}$, then $\mu K$ can be extracted by subtraction as follows (Henriques et al., 2021c):

$\mu K=K_{t}-K_{\text {aniso }}-K_{\text {iso }}$

Thus, while $K_{\mathrm{t}}$ can be accessed by conventional SDE experiments with at least two nonzero $b$-values, its decomposition in its sources requires the use of DDE preparations. Importantly, as described in Henriques et al., 2021c, the difference between the logarithm of 
260

$\log \left(\bar{E}_{D D E}\left(b_{a}, 0,0^{\circ}\right)\right)-\log \left(\bar{E}_{D D E}\left(\frac{b_{a}}{2}, \frac{b_{a}}{2}, 0^{\circ}\right)\right)=\frac{1}{12} b_{a}^{2} \bar{D}^{2} \mu K+O\left(b^{3}\right)$ $b$-value of each pair of gradients being $b_{\mathrm{a}} / 2$ can be used to directly access $\mu K$ as follows:

powder averaged signals from a SDE-like set with $b$-value $b_{\text {a }}$ and a parallel DDE set with the

263

264

265

$$
\log \left(\bar{E}_{D D E}\left(\frac{b_{a}}{2}, \frac{b_{a}}{2}, 0^{\circ}\right)\right)-\log \left(\bar{E}_{D D E}\left(\frac{b_{a}}{2}, \frac{b_{a}}{2}, 90^{\circ}\right)\right)=\frac{1}{2} b_{a}^{2} \bar{D}^{2} K_{\text {aniso }}+O\left(b^{3}\right)
$$

with the higher order terms $O\left(b^{3}\right)$ ignored in the current analysis.

\section{Participants}

Ten participants (mean age \pm one standard deviation (SD): $28.9 \pm 6.0$ years, six males) gave informed consent to participate in this study approved by the Research Ethics Committee of the University of Trento. At the time of MRI scans, all participants were healthy and had no medical history of neurological or psychiatric disorders.

\section{MRI acquisition}

Data were acquired at the Center for Mind/Brain Sciences of the University of Trento, Italy, on a 3T clinical MR scanner (MAGNETOM Prisma, Siemens Healthcare, Erlangen, Germany), with a 64-channel head-neck RF receive coil. Head motion during the acquisition was limited by the use of foam paddings optimized for head coils inside the coil. Anatomical T1-weighted (T1w) Multi-Echo MPRAGE (ME-MPRAGE, van der Kouwe et al., 2008) images were acquired with the following parameters: $\mathrm{TE}_{1} / \mathrm{TE}_{2} / \mathrm{TE}_{3} / \mathrm{TE}_{4}=1.69 / 3.55 / 5.41 / 7.27 \mathrm{~ms}, \mathrm{TR}=2530$ $\mathrm{ms}, \mathrm{TI}=1100 \mathrm{~ms}$, flip angle: $7^{\circ}, 1 \mathrm{~mm}$-isotropic resolution, matrix size: 256x256.

For the diffusion-weighted data acquisition, a prototype double-spin-echo DDE sequence was used (Fig. 1A). A double-spin-echo sequence was chosen to mitigate concomitant gradient effects (Callaghan and Komlosh, 2002; Szczepankiewicz et al., 2019b). The DDE sequence imaging parameters were: $\mathrm{TR}=5600 \mathrm{~ms}, \mathrm{TE}=127 \mathrm{~ms}$, matrix size: $84 \mathrm{x} 84$, slice thickness: 2.5 $\mathrm{mm}$, no slice gap, spatial resolution: $2.5 \mathrm{~mm}$-isotropic, 60 axial slices allowing full brain 
coverage, partial Fourier factor: 6/8, GRAPPA/SMS factors: 2/4. Four sets of DDE images were acquired according to the optimization described in Henriques et al., 2021c (Fig. 1B):

- DDE set \#1: $b_{1}=1000, b_{2}=0$, with $b_{\mathrm{t}}=1000 \mathrm{~s} / \mathrm{mm}^{2}$;

- DDE set \#2: $b_{1}=2000, b_{2}=0$, with $b_{\mathrm{t}}=2000 \mathrm{~s} / \mathrm{mm}^{2}$;

- DDE set \#3: $b_{1}=1000, b_{2}=1000$, with $b_{\mathrm{t}}=2000 \mathrm{~s} / \mathrm{mm}^{2}$ and parallel directions for the first and the second pairs of diffusion gradients $\left(\theta=0^{\circ}\right)$;

- DDE set \#4: $b_{1}=1000, b_{2}=1000$, with $b_{\mathrm{t}}=2000 \mathrm{~s} / \mathrm{mm}^{2}$ and perpendicular directions for the first and the second pairs of diffusion gradients $\left(\theta=90^{\circ}\right)$;

With $b_{\mathrm{t}}$ being the total $b$-value, i.e. including both the first and the second pairs of diffusion gradients. For all DDE sets, $\delta / \Delta / \tau_{M}=15.8 / 31.8 / 32.3 \mathrm{~ms}$. Sixty diffusion-weighted volumes were acquired per each DDE set: for sets \#1, \#2, and \#3 directions from a 3-dimensional 60point spherical 10-design were used (Hardin \& Sloane, 1996), while for set \#4, perpendicular directions from the 5-design in Jespersen et al., 2013 were used. For the phantom and all participants, one volume without diffusion gradients (hereafter referred to as " $b=0$ " volume) was added every 12 diffusion-weighted volumes, and two additional $b=0$ volumes were acquired at the beginning of set \#2 and \#4 (except for sub-01 and sub-02, where two $b=0$ volumes were included per each DDE set and TR $=5500 \mathrm{~ms}$ was used). Finally, two $b=0$ volumes with reversed phase encoding were acquired to correct the susceptibility-induced geometric distortions (see Image processing section). DDE sets were acquired in the following temporal order to guarantee the possibility of directly estimating $\mu K$ and $K_{\text {aniso }}$ in case of uncooperative participants leading to the data acquisition interruptions (see eq. 3 and 4): DDE set \#2, DDE set \#3, DDE set \#4, DDE set \#1. Two repetitions of the above listed sets were acquired, each with opposite polarity of all diffusion gradients (hereafter referred to as "positive" and "negative", Fig. 1B, bottom) to reduce potential cross-term effects with imaging gradients (Neeman et al., 1991; Lawrenz and Finsterbusch, 2011; Ianuş et al., 2018). The total acquisition time for the two repetitions was around 52 minutes.

To check for the fulfillment of the long mixing time regime assumption (Shemesh et al., 2012b; Henriques et al., 2020), twelve parallel directions were selected from the gradient scheme for DDE set \#3, and the polarity of the direction of the second pair of diffusion gradients was reversed to yield antiparallel gradients (Shemesh et al., 2012b; Henriques et al., 2020). Images corresponding to this subset of parallel and antiparallel gradient directions were acquired twice, each with opposite polarity of the diffusion gradients, followed by ten additional $b=0$ volumes 
acquired for assessing the temporal Standard Deviation (tSD) of this series and relate it to the signal difference between parallel and antiparallel images for all participants (except for sub01 and sub-02).

To verify the absence of systematic imaging artifacts, the above described protocol was additionally acquired on a brain-sized spherical isotropic phantom at the room temperature of $22^{\circ} \mathrm{C}$, filled with water doped with Nickel Sulphate $\left(\mathrm{NiSO}_{4}\left(\mathrm{H}_{2} \mathrm{O}\right)_{6}\right)$.

Image processing

324

325

326

327

328

329

330

331

332

333

334

335

336

337

338

339

340

341

342

343

344

345

346

347

Diffusion-weighted images: dMRI data were denoised with the Marchenko-Pastur PCA denoising in MRtrix v. 3.0.2 (Veraart et al., 2016), and derived noise maps were visually inspected along with denoising residuals. Data were then corrected for Gibbs ringing (Kellner et al., 2016), and the susceptibility-induced field was estimated using $b=0$ volumes with opposite phase encoding in FSL's topup (Andersson et al., 2003). Data corresponding to each set was then corrected for eddy currents and head motion in FSL's eddy (Andersson \& Sotiropoulos, 2016), by using the first acquired $b=0$ volume as reference volume for all sets, in order to get all the images aligned to the same reference volume. For DDE sets where the amplitude of the second pair of diffusion gradients was not zero (DDE set \#3, DDE set \#4), the direction of the second pair of diffusion gradients was used in eddy, motivated by the observation for a much smaller impact of the first pair of diffusion gradients on eddy currents artifacts (Mueller et al., 2017), and as reported in Yang et al., 2018 and in Fan et al., 2020. Summary metrics of the quality of each participant's images were then computed with the eddy $q c$ framework (Bastiani et al. 2019). Diffusion-weighted volumes were then concatenated according to their temporal order and corrected for signal drift (Vos et al., 2017) and bias field (Tustison et al., 2010). Phantom images underwent the same pre-processing pipeline adopted for human data. Before computing each participant's (i.e. individual) CTI maps, data were smoothed with a 3D Gaussian filter (using a Gaussian kernel with FWHM $=1.25$, scipy v. 1.6.1), and pairs of images corresponding to opposite polarities of the diffusion gradients were geometrically averaged to compensate for effects associated with potential imaging cross-terms (Neeman et al., 1991; Lawrenz and Finsterbusch, 2011; Lawrenz and Finsterbusch, 2013; Ianuş et al., 2018). Images were then directionally averaged in order to get four powder average (p.a.) images, one per each set, which were finally normalized (hereafter referred to as p.a.norm) by the mean of the $b=0$ volumes included across all the acquired DDE sets (Fig. 2A). The tSD across 
these $b=0$ volumes was computed and used to estimate a SNR map as the ratio of the mean $(\mathrm{b}=0)$ and the $\operatorname{tSD}(b=0)$ (Fig. 2B).

Anatomical images: T1w images resulting from the root mean squared combination of the four ME-MPRAGE echoes were parcellated with FreeSurfer's v. 7.1 recon-all pipeline. The following Regions Of Interest (ROIs) were considered among the areas included in the

353 FreeSurfer's parcellation for the quantitative analysis of each kurtosis source: Cerebro-Spinal 354 Fluid (CSF) in lateral ventricles, cerebral White Matter (WM), cerebellar WM (WM $\mathrm{CBM}_{\mathrm{CB}}$ ), Grey Matter (GM), cerebellar Grey Matter $\left(\mathrm{GM}_{\mathrm{CBM}}\right)$, Amygdala (AMG), Caudate $(\mathrm{Cd})$, Hippocampus (HPC), Globus Pallidus (GP), Putamen (PU), and Thalamus (TH). Linear registrations between individual skull-stripped $\mathrm{T} 1 \mathrm{w}$ images and the first pre-processed nonsmoothed $b=0$ volume were then computed in ANTs (Avants et al., 2011) and registration matrices were applied to the above listed ROIs in order to get them aligned with the CTI maps.

Registration to MNI space and MNI-space CTI analysis: In order to obtain the first, high SNR group average templates of $K_{\text {aniso }}, K_{\text {iso }}$, and $\mu K$ in the healthy human brain, CTI was fitted on across-subject averaged p.a.norm signals warped to the MNI space. This approach was preferred rather than computing the average of individual CTI maps to avoid the propagation of noise in the individual maps to the final template.

365 To normalize each participant's p.a.norm images to the MNI space, a non-linear registration between each participant's skull-stripped T1w image and the MNI $2 \mathrm{~mm}$-isotropic T1w brain atlas was computed. Finally, the resulting warp fields were applied to each p.a.norm image in combination with the linear registration matrices previously computed to align the diffusion with the anatomical data (see Anatomical images Section), to get all the p.a.norm images warped to the MNI space. All registration processes were carried out in ANTs and were visually inspected for their accuracy. No Gaussian smoothing was applied on data that were normalized to the MNI space. Once all participants' p.a.norm images were warped to the MNI space, the mean p.a.norm image across $N=8$ participants for each of the four DDE sets was computed and used for the CTI fit (hereafter referred to as MNI-space CTI analysis). Two subjects were excluded for the group MNI space analysis: i) one because of enlarged ventricles, and ii) because of unsuccessful alignment at the registration process. 
380

381

382

383

384

385

386

387

388

389

390

391

392

393

394

395

396

397

398

399

400

401

402

403

404

space CTI analysis (hereafter referred to as ROIsmNI), except for GM where voxels were included if common to at least six participants to get a more inclusive mask. Values derived from the MNI-space CTI analysis were then extracted from each $\mathrm{ROI}_{\mathrm{MNI}}$ for the quantitative analysis of the Kurtosis sources and outlier values were removed with the isoutlier function (method: Grubbs) in MatLab R2017b. Finally, in order to assess the extent of each Kurtosis source contribution to $K_{\mathrm{t}}$ maps, the ratio between each Kurtosis source and $K_{\mathrm{t}}$ was computed, and values were extracted from each ROI for further analysis. Voxels with $K \leq 0$ (any kurtosis source) were excluded from this analysis (the ratio of excluded voxels on total voxels can be found in Fig. S1).

\section{Correlation Tensor Imaging fit}

The CTI fit procedure (described in Henriques et al., 2020a, 2021) was performed using the linear least square fit function in MatLab (version R2017b). The CTI fit generates the following maps: $D_{\mathrm{t}}, K_{\mathrm{t}}, K_{\text {aniso }}, K_{\text {iso }}$, and $\mu K$ maps. For each of these maps, values were extracted from each FreeSurfer-derived ROI (see Anatomical images Section) aligned to the diffusion space. Outlier values were removed with the isoutlier function (method: Grubbs) in MatLab R2017b, and mean and standard deviation (SD) values were computed per each ROI. For the phantom, values within an eroded phantom mask excluding voxels closer than $18 \mathrm{~mm}$ to the phantom surface were extracted.

MNI-space Multiple Gaussian Component (MGC) assumption analysis

To assess the effect of neglecting $\mu K$ on the other Kurtosis sources and thus the validity of the MGC assumption, similarly to Henriques et al., 2021c, $K_{\text {aniso }}$ and $K_{\text {iso }}$ were derived under the MGC assumption according to the tensor-valued information of the diffusion MRI acquisitions (Westin et al., 2014; Szczepankiewicz et al., 2019a). p.a.norm images used for the MNI-space CTI analysis (thus only using axial tensor-valued experiments), were fitted with eq. (5):

$\log \left(\bar{E}_{M G C}\left(b, b_{\Delta}\right)\right)=-b_{t} \bar{D}+\frac{1}{6} b_{t}^{2} \bar{D}^{2} K_{i s o}^{M G C}+\frac{1}{6} b_{t}^{2} b_{\Delta}^{2} \bar{D}^{2} K_{\text {aniso }}^{M G C}+O\left(b^{3}\right)$

with $b_{\mathrm{t}}$ being the cumulative $b$-value (i.e. considering both pairs of diffusion gradients), and $b_{\Delta}$ being 1 for DDE sets \#1-3 and -1/2 for DDE set \#4. 
408 In order to assess effects of imaging cross-terms, p.a.norm was computed separately on data 409 corresponding to each polarity of the diffusion gradients (i.e. "positive" and "negative", Fig.

$4101 \mathrm{~B}$, bottom). p.a.norm images then underwent the same registration pipeline described in the 411 Registration to MNI space and MNI-space CTI analysis paragraph. $\mu \mathrm{K}$ values were extracted 412 from each ROI for their quantitative analysis.

413 Data and code availability

414 Data and code used for data analysis are available via request to the authors, with the need of a 415 formal sharing agreement. 
A) $b=0$ volume and ROIs

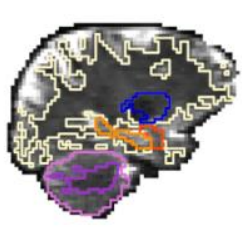

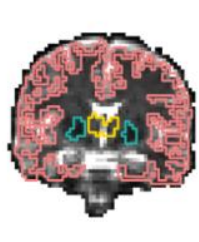

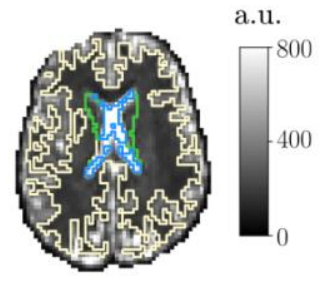

B) Signal to Noise Ratio

C) Normalized powder averaged images
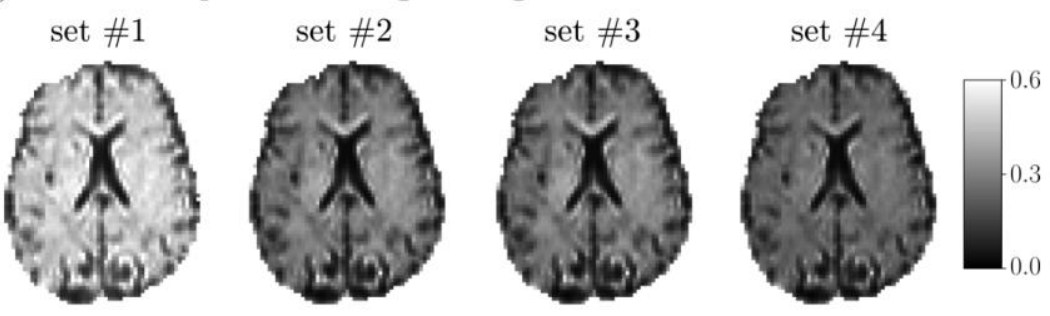

D) Long mixing time regime verification $E_{p . a .}\left(0^{\circ}\right)-E_{p . a .}\left(180^{\circ}\right)$ $E_{p . a .}\left(0^{\circ}\right)-E_{p . a .}\left(180^{\circ}\right)$

E) Phantom assessments
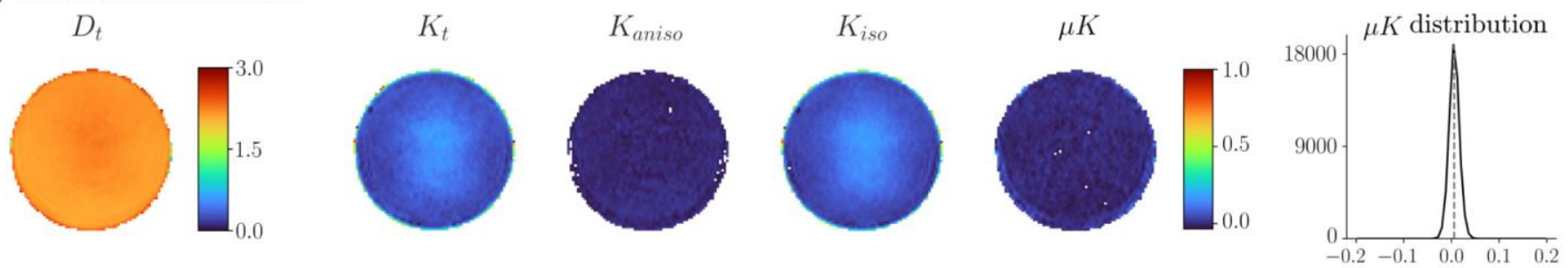

Fig. 2, Data quality. All brain images come from the same slices of a representative subject. A) $b=0$ volume with overlaid borders of Regions of Interest (ROIs) used in this study. Lateral view: yellow:

418 white matter; blue: putamen; orange: hippocampus; red: amygdala; light violet: cerebellar cortex; violet: 419 cerebellar white matter. Coronal view: pink: cerebral cortex; yellow: thalamus; green: pallidum. Axial view: yellow: white matter; green: caudate; light blue: lateral ventricles. B) Lateral, coronal, and axial view of the Signal to Noise Ratio (SNR) map. C) Powder (i.e. directionally) averaged images per each set normalized by the mean $b=0$ volume (p.a.norm). D) Left: map of the difference between parallel and antiparallel p.a. images at $b_{\mathrm{t}}=2000 \mathrm{~s} / \mathrm{mm}^{2}$ with the brain border marked in red. Right: distribution of signal differences between the parallel and antiparallel p.a. images (black), with the distribution of the temporal standard deviation values across $b=0$ shown in light blue. E) CTI maps derived from the phantom experiment, and distribution of $\mu K$ values within the phantom. Human data underwent the preprocessing pipeline described in the Materials and Methods section, and are shown before the application of the Gaussian smoothing. 


\section{Results}

\section{Data quality}

431 Fig. 2A shows an example $\mathrm{b}=0$ volume with overlaid FreeSurfer-derived ROIs aligned to the

432 diffusion space for a representative subject. Across subjects, mean SNR \pm one SD within the

433 brain was $20 \pm 1.35$. The SNR map and p.a.norm images of non-smoothed data for a 434 representative subject are shown in Fig. 2B and C, respectively. The difference between parallel 435 and antiparallel gradient pairs designed to test for the long mixing time regime, is shown in Fig.

436 2D: the signal differences did not reveal visible anatomical structures for all sampled directions, 437 and were on the order of the tSD estimated on $b=0$ volumes in the same series.

\section{$438 \quad$ Phantom assessments}

439 CTI maps from the phantom (designed to ensure no systematic effects are observed) are shown in Fig. 2E (before Gaussian smoothing). Mean \pm one SD values per each CTI map were: $D_{\mathrm{t}}$ : $2.16 \pm 0.11 ; K_{\mathrm{t}}: 0.09 \pm 0.04 ; K_{\text {aniso }}: 0.00 \pm 0.01 ; K_{\text {iso }}: 0.08 \pm 0.04 ; \mu K: 0.01 \pm 0.01 ;$ (see also 442 Table 1).

\begin{tabular}{|c|c|c|c|c|c|c|c|c|c|c|c|c|}
\hline \multirow[t]{2}{*}{ Map } & \multicolumn{11}{|c|}{$\begin{array}{c}\text { Human data } \\
\text { Regions of Interest (ROIs) }\end{array}$} & \multirow[t]{2}{*}{ Phantom } \\
\hline & $\mathrm{CSF}$ & WM & $\mathrm{WM}_{\mathrm{CBM}}$ & GM & $\mathrm{GM}_{\mathrm{CBM}}$ & AMG & $\mathrm{Cd}$ & $\mathrm{HPC}$ & GP & PU & $\mathrm{TH}$ & \\
\hline$K_{t}$ & $\begin{array}{c}0.49 \pm \\
0.04\end{array}$ & $\begin{array}{l}1.04 \pm \\
0.02\end{array}$ & $\begin{array}{l}1.23 \pm \\
0.06\end{array}$ & $\begin{array}{c}0.78 \pm \\
0.01\end{array}$ & $\begin{array}{c}0.97 \pm \\
0.04\end{array}$ & $\begin{array}{c}0.82 \pm \\
0.04\end{array}$ & $\begin{array}{c}0.83 \pm \\
0.06\end{array}$ & $\begin{array}{c}0.86 \pm \\
0.03\end{array}$ & $\begin{array}{c}1.54 \pm \\
0.08\end{array}$ & $\begin{array}{c}1.08 \pm \\
0.05\end{array}$ & $\begin{array}{l}1.1 \pm \\
0.07\end{array}$ & $\begin{array}{c}0.09 \pm \\
0.04\end{array}$ \\
\hline$K_{\text {aniso }}$ & $\begin{array}{c}0.02 \pm \\
0.01\end{array}$ & $\begin{array}{c}0.40 \pm \\
0.03\end{array}$ & $\begin{array}{c}0.48 \pm \\
0.03\end{array}$ & $\begin{array}{c}0.08 \pm \\
0.01\end{array}$ & $\begin{array}{c}0.10 \pm \\
0.02\end{array}$ & $\begin{array}{c}0.07 \pm \\
0.01\end{array}$ & $\begin{array}{c}0.06 \pm \\
0.02\end{array}$ & $\begin{array}{c}0.06 \pm \\
0.01\end{array}$ & $\begin{array}{c}0.26 \pm \\
0.08\end{array}$ & $\begin{array}{c}0.15 \pm \\
0.03\end{array}$ & $\begin{array}{c}0.21 \pm \\
0.02\end{array}$ & $\begin{array}{c}0.00 \pm \\
0.01\end{array}$ \\
\hline$K_{\text {iso }}$ & $\begin{array}{c}0.46 \pm \\
0.03\end{array}$ & $\begin{array}{c}0.47 \pm \\
0.04\end{array}$ & $\begin{array}{c}0.60 \pm \\
0.07\end{array}$ & $\begin{array}{c}0.57 \pm \\
0.02\end{array}$ & $\begin{array}{c}0.70 \pm \\
0.05\end{array}$ & $\begin{array}{c}0.60 \pm \\
0.04\end{array}$ & $\begin{array}{c}0.65 \pm \\
0.05\end{array}$ & $\begin{array}{c}0.69 \pm \\
0.02\end{array}$ & $\begin{array}{c}1.13 \pm \\
0.13\end{array}$ & $\begin{array}{c}0.73 \pm \\
0.08\end{array}$ & $\begin{array}{c}0.80 \pm \\
0.08\end{array}$ & $\begin{array}{c}0.08 \pm \\
0.04\end{array}$ \\
\hline$\mu K$ & $\begin{array}{c}0.01 \pm \\
0.01\end{array}$ & $\begin{array}{c}0.16 \pm \\
0.01\end{array}$ & $\begin{array}{c}0.15 \pm \\
0.03\end{array}$ & $\begin{array}{c}0.13 \pm \\
0.02\end{array}$ & $\begin{array}{c}0.18 \pm \\
0.02\end{array}$ & $\begin{array}{c}0.16 \pm \\
0.01\end{array}$ & $\begin{array}{c}0.12 \pm \\
0.02\end{array}$ & $\begin{array}{c}0.10 \pm \\
0.01\end{array}$ & $\begin{array}{c}0.15 \pm \\
0.04\end{array}$ & $\begin{array}{c}0.20 \pm \\
0.02\end{array}$ & $\begin{array}{c}0.10 \pm \\
0.02\end{array}$ & $\begin{array}{c}0.01 \pm \\
0.01\end{array}$ \\
\hline
\end{tabular}
one SD. For human data, the SD was computed across mean values of each participant's data. For phantom data, the SD was computed spatially, i.e. across all included phantom voxels. Abbreviations: 
447 GM: Grey Matter; GM

448 Hippocampus; GP: Globus Pallidus; PU: Putamen; TH: Thalamus.

\section{Individual CTI maps analysis}

450 All data was successfully acquired without any interruption due to uncooperative participants. 451 Individual CTI-derived maps are shown in Fig. 3. Between subjects, maps consistently showed 452 larger $K_{\text {aniso }}$ values for white matter areas, larger $K_{\text {iso }}$ values at the interface between tissues, 453 and $\mu K$ values that were centered on zero for CSF and non-vanishing positive for both grey and 454 white matter. Across-subject mean \pm one SD Kurtosis values of CTI-derived maps are listed 455 per each ROI in Table 1. Distributions of Kurtosis values on individual maps per each ROI can 456 be found on Fig. S2.

\section{MNI-space CTI analysis}

458 CTI maps computed on across-subjects averaged p.a.norm images are shown in Fig. 4A, along 459 with distributions of values in each ROI (Fig. 4B). Consistently with individual-level maps, $460 K_{\text {aniso }}$ was larger in WM regions, and $K_{\text {iso }}$ was larger in regions that likely present higher degree 461 of partial volume with CSF (regions near ventricles and edge of cortex); $\mu K$ showed zero462 centered values in CSF, but non-vanishing positive values both in grey and white matter (Table 463 2, voxels identified as outliers and excluded from the plotted distributions and quantitative 464 analyses were $<3 \%$ for all ROIs and maps except for GP $K_{\text {aniso, }}$, where excluded voxels 465 represented $3.64 \%$ of total voxels; see Table S1 for number of excluded voxels and percentages 466 on total voxels for all ROIs and maps). Fig. 5A-C shows maps of the ratio between each 467 Kurtosis source and $K_{\mathrm{t}}$, along with barplots showing the Kurtosis components per each ROI 468 (Fig. 5D): $K_{\text {iso }}$ appears to be the largest Kurtosis source for all tissues except white matter regions, with $\mu K$ accounting for 8-20\% of $K_{\mathrm{t}}$ in all ROIs except for CSF. 
bioRxiv preprint doi: https:/doi.org/10.1101/2021.11.02.466950; this version posted November 4 2021. The copyright holder for this preprint (which was not certified by peer review) is the author/funder, who has granted bioRxiv a license to display the preprint in perpetuity. It is made available under aCC-BY-NC-ND 4.0 International license.

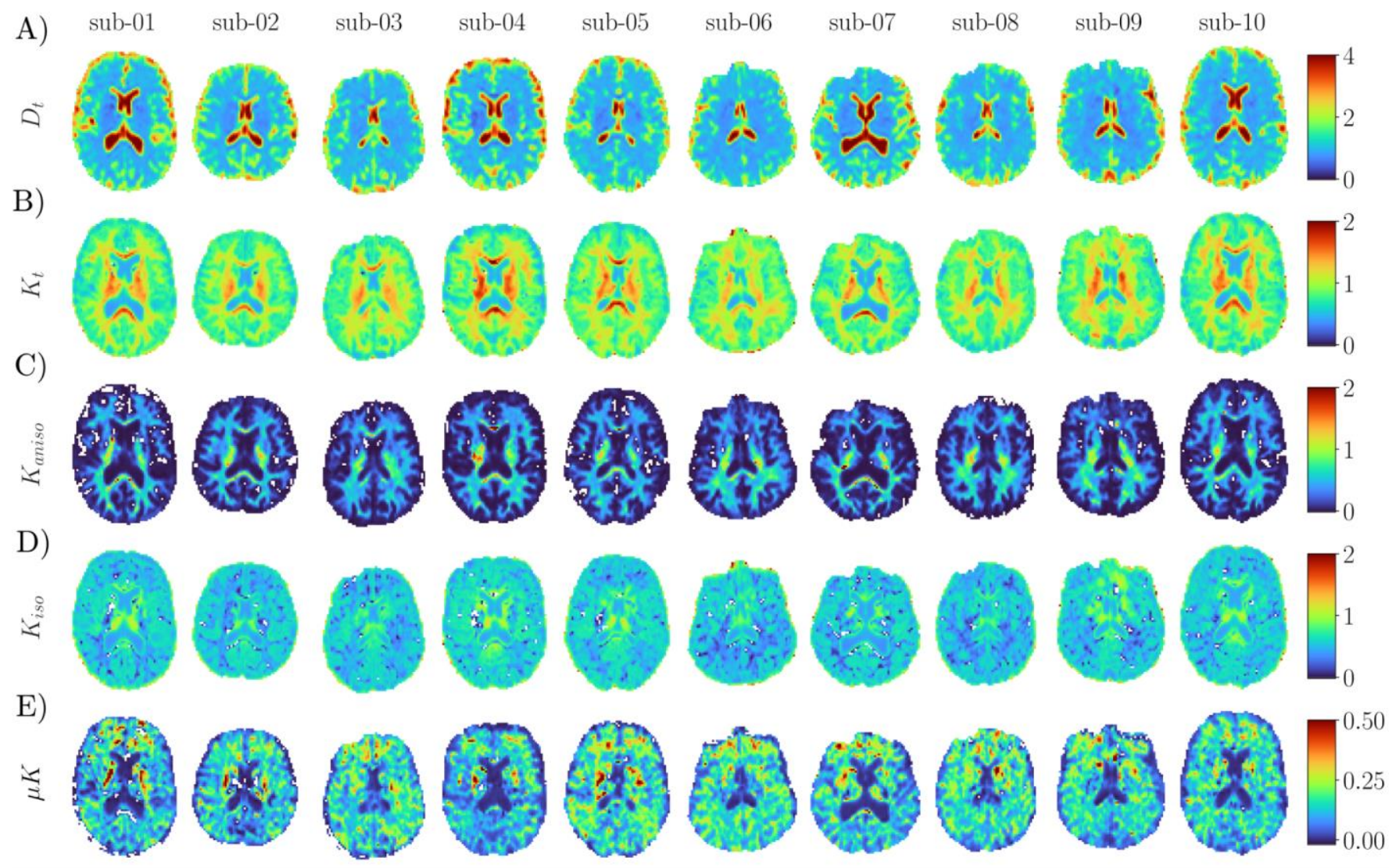

470 Fig. 3, Individual CTI-derived maps in native space. A) $D_{\mathrm{t}}$ maps; B) $K_{\mathrm{t}}$ maps; C) $K_{\text {aniso }}$ maps; D) $K_{\text {iso }}$ 471 maps. White voxels within the brain correspond to negative $K_{\text {iso }}$ values, possibly associated with noise 472 effects; a discussion on negative kurtosis values can be found e.g. in Henriques et al., 2021d; E) $\mu K$ 473 maps. Note the different ranges in the colorbars for different CTI metrics.

474 MNI-space CTI vs. MGC analysis

475 Fig. 6 shows the relationship between total, anisotropic and isotropic kurtosis estimates derived 476 from CTI and the corresponding ones estimated under the MGC assumption, which neglects $477 \mu K$. For any of these metrics, a bias between the two approaches will be seen as a distribution 478 that departs from the diagonal. Relative to CTI, the MGC approach leads to lower $K_{\mathrm{t}}$ in voxels 479 with high $\mu K$. The opposite pattern was observed for $K_{\text {aniso }}$ and $K_{\text {iso, }}$, where estimates derived 480 under the MGC assumption were overestimated compared to CTI-derived values, with larger 481 deviations being associated to larger $\mu K$ values. 
A)
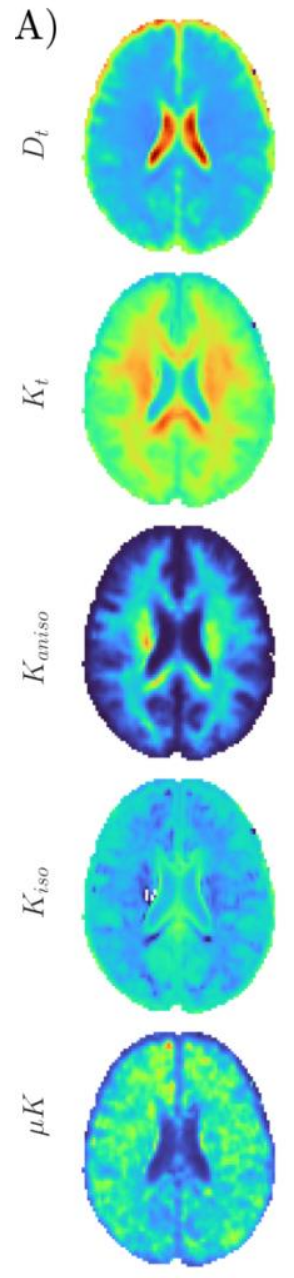
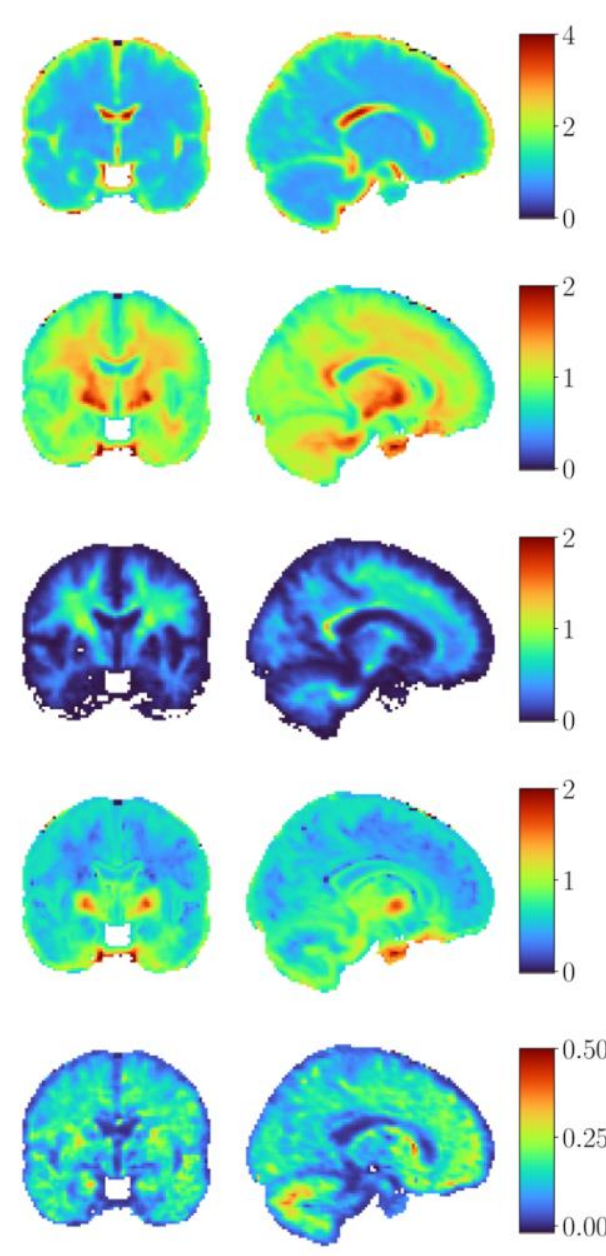

B)

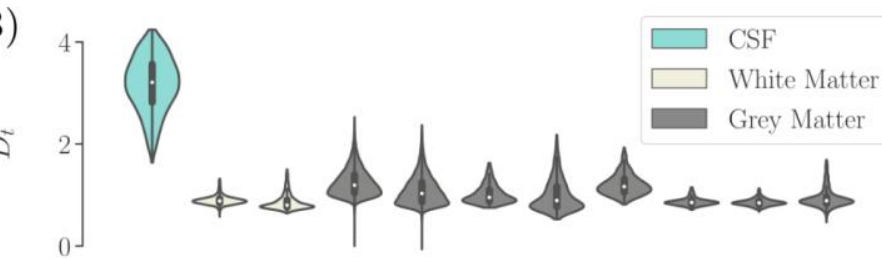

$=1$
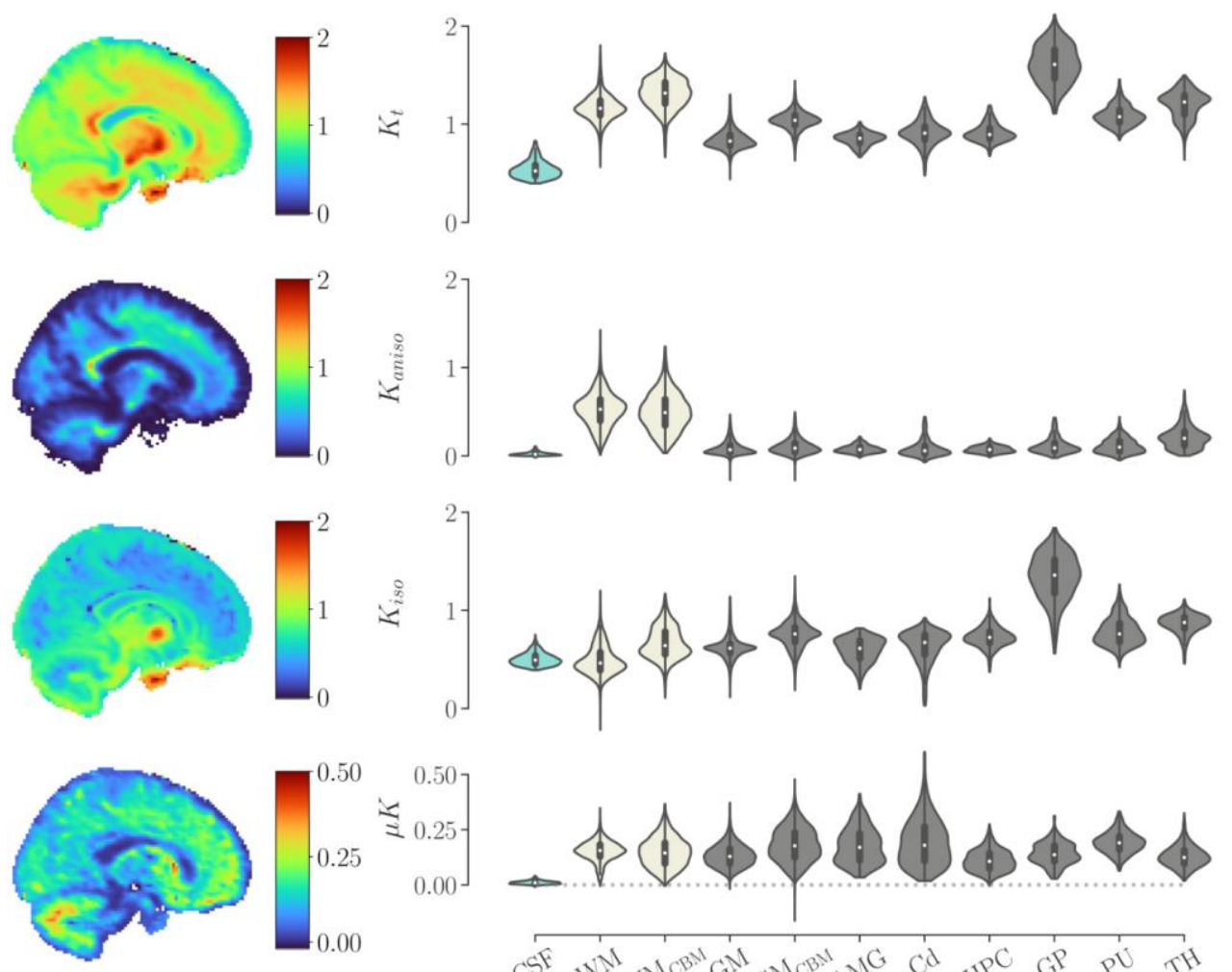

483 CTI analysis). A) Axial, coronal, and lateral views of $D_{\mathrm{t}}, K_{\mathrm{t}}, K_{\text {aniso }}, K_{\mathrm{iso}}$, and $\mu K$ maps derived by fitting

484 the averaged data of $N=8$ subjects, previously normalized to the $2 \mathrm{~mm}$ MNI atlas. Note the different 485 ranges in the colorbars for different CTI metrics. B) Distributions of values per each CTI metric in each 486 Region of Interest (ROI) considered in this study. CSF: Cerebrospinal fluid in lateral ventricles; WM: 487 White Matter; WM Свм: Cerebellar White Matter; GM: Grey Matter; GM Свм: Cerebellar Grey Matter; 488 AMG: Amygdala; Cd: Caudate; HPC: Hippocampus; GP: Globus Pallidus; PU: Putamen; TH: 489 Thalamus.

491 Fig. 7 shows CTI-derived maps from the averaged data corresponding to either both or single repetitions of the CTI acquisition. In comparison with maps estimated from two repetitions 
bioRxiv preprint doi: https:/doi org/10.1101/2021.11.02466950; this version posted November 4,2021 . The copyright holder for this preprint (which was not certified by peer review) is the author/funder, who has granted bioRxiv a license to display the preprint in perpetuity. It is made available under aCC-BY-NC-ND 4.0 International license.

\begin{tabular}{|c|c|c|c|c|c|c|c|c|c|c|c|}
\hline \multirow[t]{2}{*}{ Map } & \multicolumn{11}{|c|}{$\begin{array}{c}\text { Human group average template data: } M N I \text {-space CTI analysis } \\
\text { Regions of Interest (ROIs) }\end{array}$} \\
\hline & CSF & WM & $\mathrm{WM}_{\mathrm{CBM}}$ & GM & $\mathrm{GM}_{\mathrm{CBM}}$ & AMG & $\mathrm{Cd}$ & HPC & GP & PU & $\mathrm{TH}$ \\
\hline$K_{\mathrm{t}}$ & $\begin{array}{c}0.54 \pm \\
0.09\end{array}$ & $\begin{array}{c}1.17 \pm \\
0.13\end{array}$ & $\begin{array}{c}1.31 \pm \\
0.15\end{array}$ & $\begin{array}{c}0.84 \pm \\
0.09\end{array}$ & $\begin{array}{c}1.04 \pm \\
0.09\end{array}$ & $\begin{array}{c}0.85 \pm \\
0.07\end{array}$ & $\begin{array}{c}0.91 \pm \\
0.12\end{array}$ & $\begin{array}{c}0.91 \pm \\
0.09\end{array}$ & $\begin{array}{c}1.61 \pm \\
0.19\end{array}$ & $\begin{array}{c}1.09 \pm \\
0.1\end{array}$ & $\begin{array}{c}1.20 \pm \\
0.14\end{array}$ \\
\hline$K_{\text {aniso }}$ & $\begin{array}{c}0.02 \pm \\
0.02\end{array}$ & $\begin{array}{c}0.52 \pm \\
0.19\end{array}$ & $\begin{array}{c}0.50 \pm \\
0.21\end{array}$ & $\begin{array}{c}0.90 \pm \\
0.08\end{array}$ & $\begin{array}{c}0.10 \pm \\
0.09\end{array}$ & $\begin{array}{c}0.08 \pm \\
0.04\end{array}$ & $\begin{array}{c}0.08 \pm \\
0.09\end{array}$ & $\begin{array}{c}0.07 \pm \\
0.04\end{array}$ & $\begin{array}{c}0.12 \pm \\
0.09\end{array}$ & $\begin{array}{c}0.11 \pm \\
0.08\end{array}$ & $\begin{array}{c}0.21 \pm \\
0.13\end{array}$ \\
\hline $\boldsymbol{K}_{\text {aniso }} /$ & & & & & & & & & & & \\
\hline $\begin{array}{c}\boldsymbol{K}_{\mathbf{t}} \\
\% \text { ratio }\end{array}$ & $\begin{array}{c}3.5 \\
(3.9)\end{array}$ & $\begin{array}{c}45.5 \\
(17.2)\end{array}$ & $\begin{array}{c}37.5 \\
(18.6)\end{array}$ & $\begin{array}{c}8.7 \\
(9.6)\end{array}$ & $\begin{array}{c}9.0 \\
(8.7)\end{array}$ & $\begin{array}{c}8.3 \\
(6.9)\end{array}$ & $\begin{array}{c}7.5 \\
(9.9)\end{array}$ & $\begin{array}{c}8.0 \\
(5.8)\end{array}$ & $\begin{array}{c}5.8 \\
(6.5)\end{array}$ & $\begin{array}{c}9.7 \\
(11.6)\end{array}$ & $\begin{array}{c}16.4 \\
(11.3)\end{array}$ \\
\hline$K_{\text {iso }}$ & $\begin{array}{c}0.50 \pm \\
0.06\end{array}$ & $\begin{array}{c}0.49 \pm \\
0.15\end{array}$ & $\begin{array}{c}0.67 \pm \\
0.16\end{array}$ & $\begin{array}{c}0.62 \pm \\
0.11\end{array}$ & $\begin{array}{c}0.75 \pm \\
0.13\end{array}$ & $\begin{array}{c}0.60 \pm \\
0.12\end{array}$ & $\begin{array}{c}0.63 \pm \\
0.16\end{array}$ & $\begin{array}{c}0.73 \pm \\
0.1\end{array}$ & $\begin{array}{c}1.34 \pm \\
0.23\end{array}$ & $\begin{array}{c}0.78 \pm \\
0.14\end{array}$ & $\begin{array}{c}0.86 \pm \\
0.1\end{array}$ \\
\hline $\begin{array}{l}\boldsymbol{K}_{\text {iso }} / \boldsymbol{K}_{\mathbf{t}} \\
\% \text { ratio }\end{array}$ & $\begin{array}{l}94.5 \\
(4.9)\end{array}$ & $\begin{array}{c}40.4 \\
(17.5)\end{array}$ & $\begin{array}{c}48.7 \\
(20.1)\end{array}$ & $\begin{array}{c}75.3 \\
(15.5)\end{array}$ & $\begin{array}{c}73.8 \\
(18.1)\end{array}$ & $\begin{array}{l}70.3 \\
(18)\end{array}$ & $\begin{array}{c}70.8 \\
(23.1)\end{array}$ & $\begin{array}{c}80.2 \\
(10.7)\end{array}$ & $\begin{array}{l}85.7 \\
(6.5)\end{array}$ & $\begin{array}{c}71.7 \\
(12.8)\end{array}$ & $\begin{array}{c}73.0 \\
(11.6)\end{array}$ \\
\hline$\mu K$ & $\begin{array}{c}0.01 \pm \\
0.01\end{array}$ & $\begin{array}{c}0.15 \pm \\
0.05\end{array}$ & $\begin{array}{c}0.15 \pm \\
0.07\end{array}$ & $\begin{array}{c}0.13 \pm \\
0.05\end{array}$ & $\begin{array}{c}0.18 \pm \\
0.08\end{array}$ & $\begin{array}{c}0.18 \pm \\
0.08\end{array}$ & $\begin{array}{c}0.19 \pm \\
0.1\end{array}$ & $\begin{array}{c}0.11 \pm \\
0.05\end{array}$ & $\begin{array}{c}0.14 \pm \\
0.05\end{array}$ & $\begin{array}{c}0.19 \pm \\
0.05\end{array}$ & $\begin{array}{c}0.13 \pm \\
0.05\end{array}$ \\
\hline $\begin{array}{l}\boldsymbol{\mu} \boldsymbol{K} / \boldsymbol{K}_{\mathbf{t}} \\
\% \text { ratio }\end{array}$ & $\begin{array}{c}1.9 \\
(1.6)\end{array}$ & $\begin{array}{l}13.2 \\
(5.1)\end{array}$ & $\begin{array}{l}10.8 \\
(7.5)\end{array}$ & $\begin{array}{l}15.0 \\
(7.5)\end{array}$ & $\begin{array}{c}17.0 \\
(10.1)\end{array}$ & $\begin{array}{c}19.6 \\
(15.9)\end{array}$ & $\begin{array}{c}17.9 \\
(16.5)\end{array}$ & $\begin{array}{l}11.6 \\
(7.5)\end{array}$ & $\begin{array}{l}8.2 \\
\text { (4) }\end{array}$ & $\begin{array}{l}17.3 \\
(6.4)\end{array}$ & $\begin{array}{l}10.1 \\
(5.1)\end{array}$ \\
\hline
\end{tabular}

Table 2, Kurtosis sources values for CTI maps derived from the average of data from $N=8$

correspond to mean \pm one SD. Values of percent ratio between each Kurtosis source and $K_{\mathrm{t}}$ correspond to median (interquartile range, IQR). The SDs and the IQRs were computed spatially, i.e. across all included ROI voxels. Abbreviations: CSF: Cerebrospinal fluid in lateral ventricles; WM: White Matter; WM $_{\text {Свм}}$ : Cerebellar White Matter; GM: Grey Matter; GM Amygdala; Cd: Caudate; HPC: Hippocampus; GP: Globus Pallidus; PU: Putamen; TH: Thalamus.

500 (corresponding to a $\sim 52$ minutes-long acquisition), maps computed from single repetitions 501 suggest that there is no systematic bias when considering only one polarity of the diffusion 502 gradients. Distributions of $\mu K$ values derived from single repetitions per each ROI can be found on Fig. S3. 
A) $K_{\text {aniso }} / K_{t}$
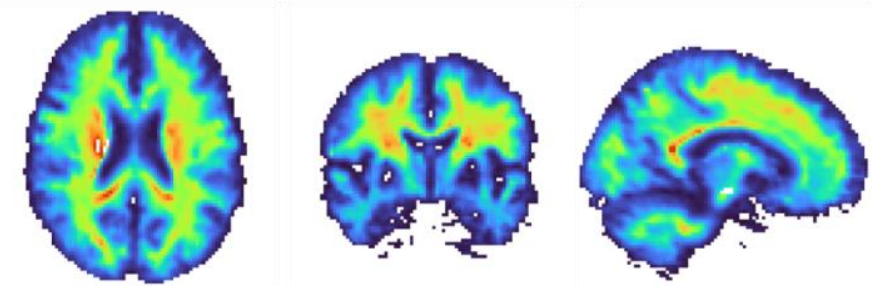

B) $K_{i s o} / K_{t}$
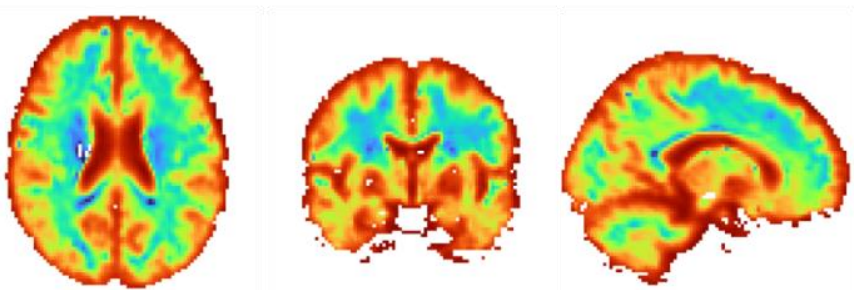

C) $\mu K / K_{t}$
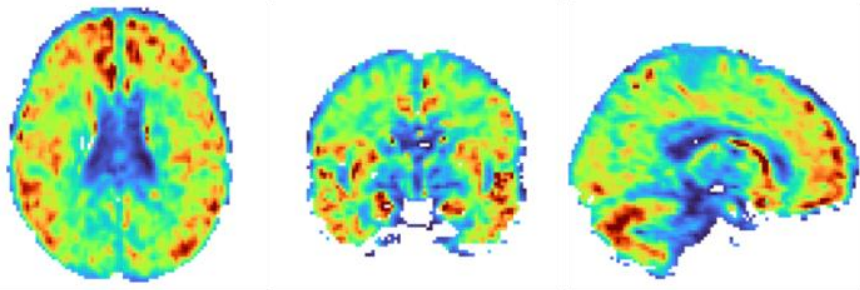


D) $K_{\text {aniso }}, K_{\text {iso }}, \mu K$ ratio on $K_{t}$
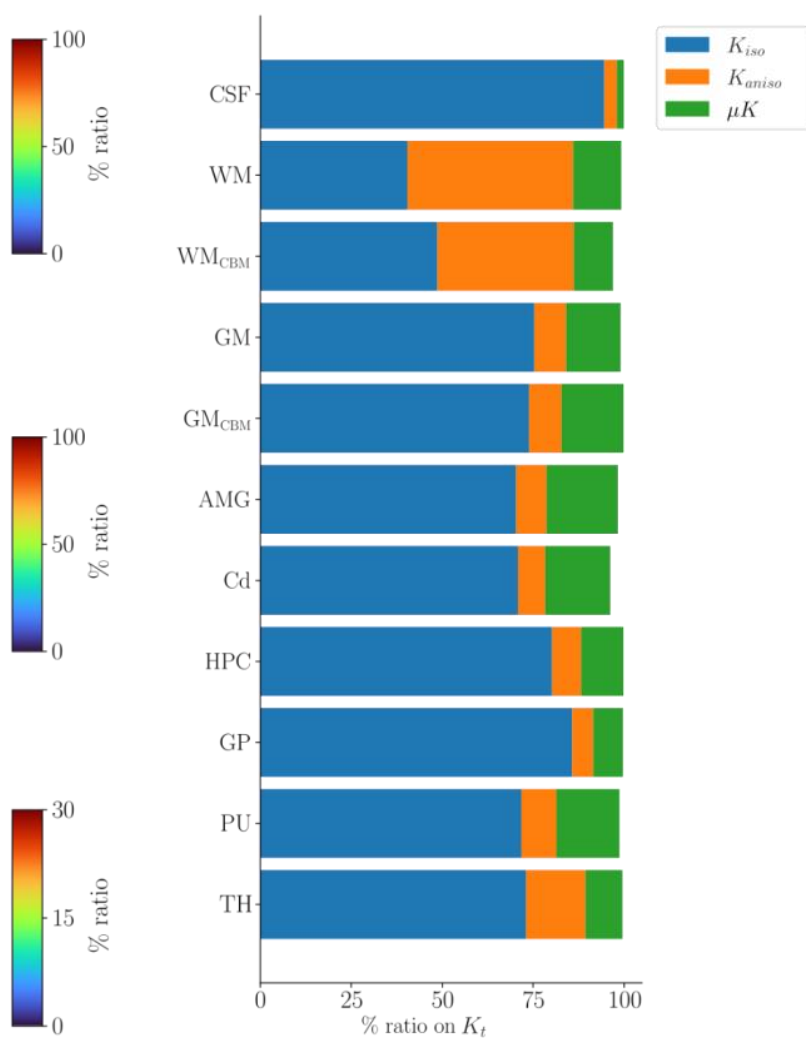

504 Fig. 5, Ratio maps of each Kurtosis source on total Kurtosis. CTI maps derived from the average of

505 data from $N=8$ subjects in MNI space. Axial, coronal, and lateral views of A) $K_{\text {aniso }} / K_{\mathrm{t}}$ ratio, B) $K_{\text {iso }} / K_{\mathrm{t}}$

506 ratio, and C) $\mu K / K_{\mathrm{t}}$ ratio. Note the different ranges in the colorbars for different ratios. D) Barplot

507 showing median percent ratio per each Kurtosis source per each Region of Interest (ROI) considered in

508 this study. CSF: Cerebrospinal fluid in lateral ventricles; WM: White Matter; $\mathrm{WM}_{\mathrm{CBM}}$ : Cerebellar White

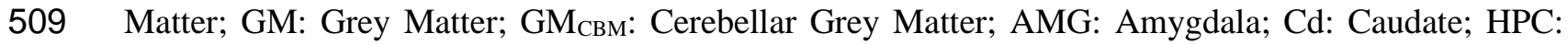

510 Hippocampus; GP: Globus Pallidus; PU: Putamen; TH: Thalamus. 

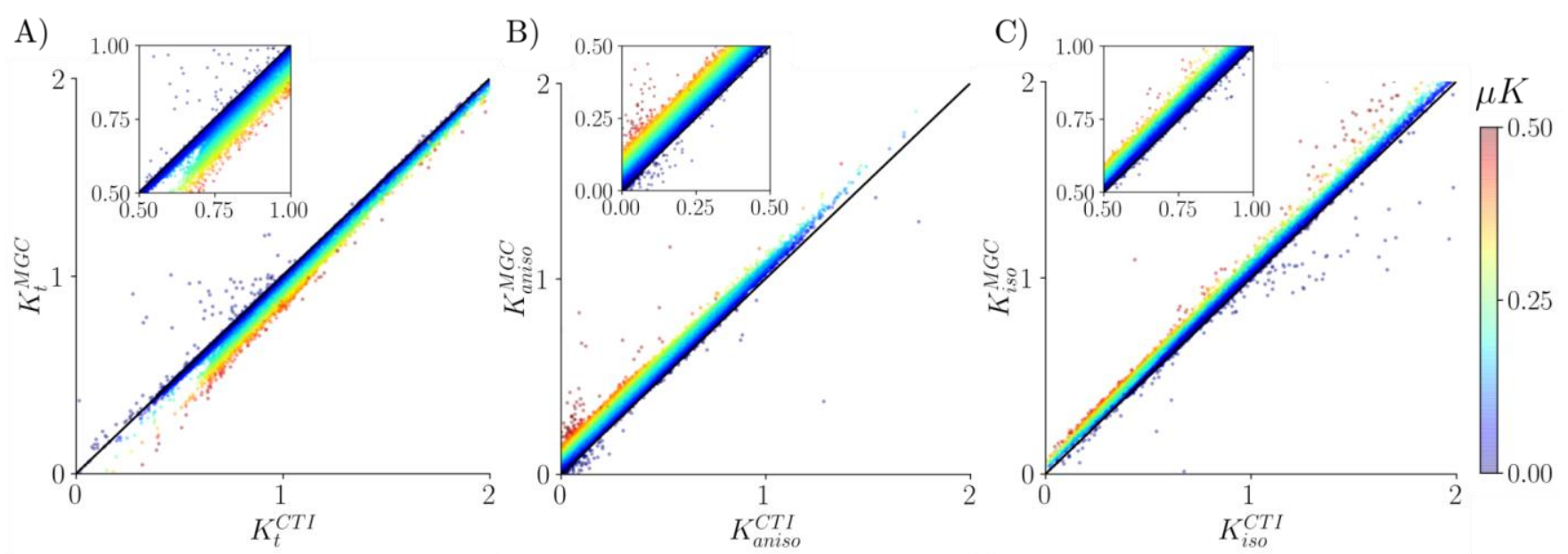

511 Fig. 6, Effect of neglecting $\boldsymbol{\mu} \boldsymbol{K}$ on $\boldsymbol{K}_{\mathrm{t}}, \boldsymbol{K}_{\text {aniso, }}$ and $\boldsymbol{K}_{\text {iso. }}$ CTI- and MGC-derived maps were estimated on

512 the average of data from $N=8$ subjects in MNI space. A) CTI- vs. MGC-derived $K_{\mathrm{t}}$. B) CTI- vs. MGC-

513 derived $K_{\text {aniso. }}$ C) CTI- vs. MGC-derived $K_{\text {iso. }}$. Points are color-coded according to their $\mu K$ value, inset 514 plots are added for better visibility. 
bioRxiv preprint doi: https:/doi.org/10.1101/2021.11.02.466950; this version posted November 4, 2021. The copyright holder for this preprint (which was not certified by peer review) is the author/funder, who has granted bioRxiv a license to display the preprint in perpetuity. It is made available under aCC-BY-NC-ND 4.0 International license.

A) Positive and negative polarity, geometrical average TA: 52 minutes


B) Positive polarity

$$
\text { TA: } \sim 26 \text { minutes }
$$
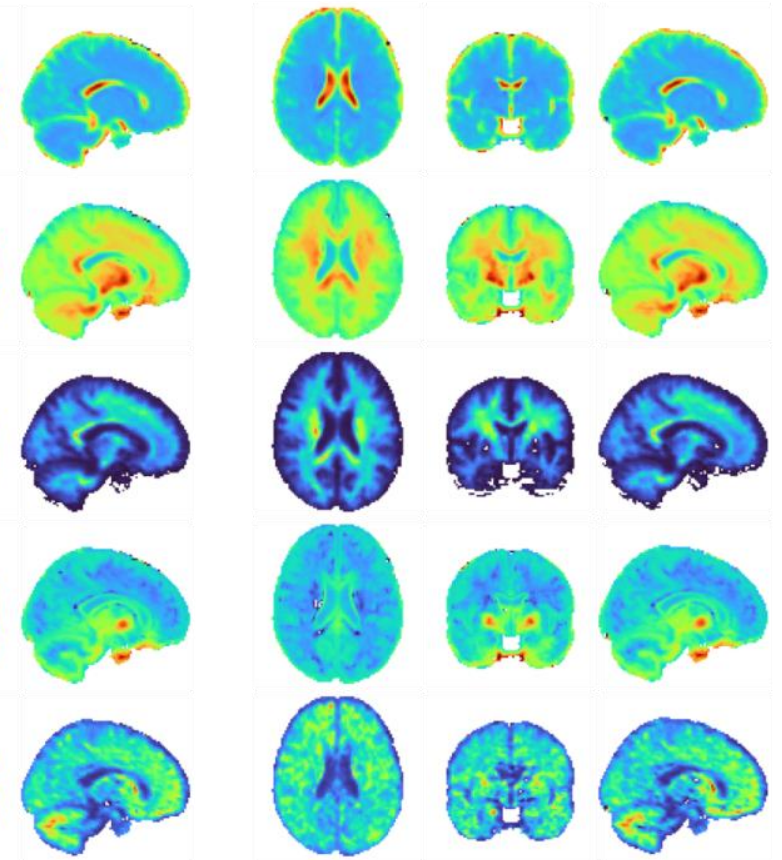

C) Negative polarity

TA: 26 minutes
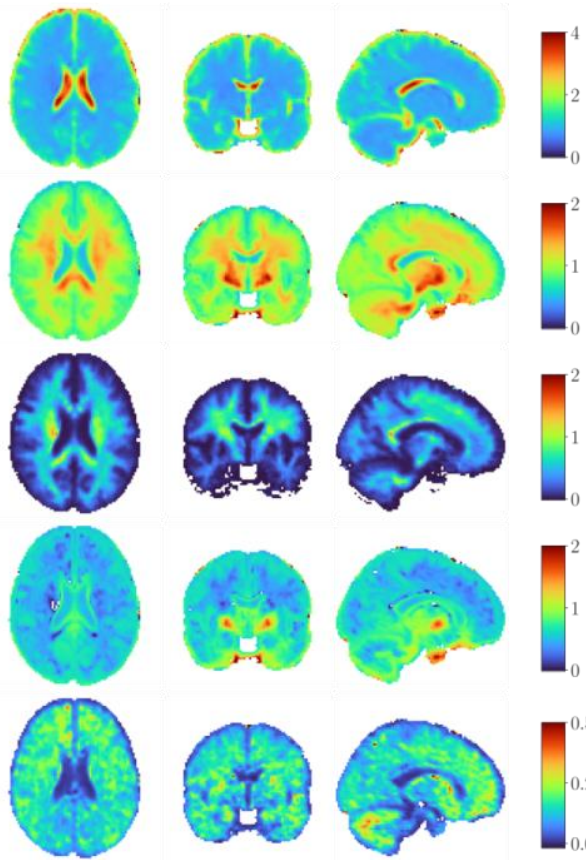

515 Fig. 7, Effect of reducing the acquisition time by $50 \%$ on CTI-derived maps. CTI-derived maps

516 were estimated on the average of data from $N=8$ subjects in MNI space. Axial, coronal, and lateral

517 views of A) CTI-derived maps estimated from the geometrical average of two repetitions, each acquired

518 with opposite polarity of the diffusion gradients (here referred to as "positive" and "negative", Neeman

519 et al., 1991; Ianuş et al., 2018), yielding a 52-minutes long acquisition; B) CTI-derived maps estimated

520 from only one repetition corresponding to the "positive" polarity of the diffusion gradients; C) CTI-

521 derived maps estimated from only one repetition corresponding to the "negative" polarity of the

522 diffusion gradients. Note the different ranges in the colorbars for different CTI metrics. Acquisition

523 time corresponding to only one repetition (panels B and C) was 26 minutes. TA: Acquisition time. 


\section{Discussion}

525 Disentangling kurtosis sources in biological systems is attracting increasing interest given the

526

527

528

529

530

531

532

533

534

535

536

537

538

539

540

541

542

543

544

545

546

547

548

549

550

551

552

553

potential to increase specificity and potentially provide novel non-invasive quantitative markers of microstructural properties in vivo. The recently-proposed CTI framework is designed to resolve anisotropic, isotropic, and microscopic kurtosis sources, which indeed provided much insight in preclinical imaging. In this work, we have extended the CTI methodology towards human imaging on a clinical 3T MRI system and aimed to investigate the kurtosis sources in healthy volunteers. Our main findings revealed not only the anisotropic and isotropic kurtosis sources in the human brain, but also clearly showed non-vanishing positive microscopic kurtosis contributions accounting for $8-20 \%$ of the total diffusional kurtosis, larger in white matter than in gray matter, and vanishing in the ventricles. Consistently with this, we also show that MGC-based approaches for kurtosis source separation are biased when neglecting microscopic kurtosis. The results of the present study pave the way towards quantifying kurtosis sources more precisely and accurately, investigating them in different types of disease. Our findings motivate further developments to accelerate data acquisition to make human brain CTI more compatible with clinical scan times.

\section{Value of separating kurtosis sources using the CTI methodology}

Decomposing the total kurtosis into its underlying sources using CTI revealed specific complementary contrasts for each kurtosis component both in individual maps and the group average template. For instance, $K_{\mathrm{t}}$ maps show elevated values in regions where partial volume effects are expected, such as around the lateral ventricles, and more in general at tissue interfaces; the separation in sources, however, allows to disentangle the relative contributions arising from each kurtosis source. In particular, the CTI method enables the evaluation of $\mu K$ which cannot be obtained from other tensor-valued approaches. More specifically, with the diffusion times adopted in the current study, positive $\mu K$ values were observed both for grey and white matter for both individual maps and the group average template, suggesting that the kurtosis component arising from non-Gaussian effects might be ubiquitous in the cerebral tissue. Interestingly, the quantitative analysis of both individual maps and the group average template also showed large $K_{\mathrm{t}}$ values for the globus pallidus, and the separation of $K_{\mathrm{t}}$ in its sources revealed that such elevated kurtosis values were mostly driven by high $K_{\text {iso. }}$ The short 
554

555

556

557

558

559

560

561

562

563

564

565

566

567

568

569

570

571

572

573

574

575

576

577

578

579

580

581

582

583

584

585

586

$T_{2}$ characterizing this structure in combination with the longer echo time needed for the DDE preparation, yields a low SNR, which is likely the root of the observed high $K_{\text {iso }}$ values (Jensen \& Helpern, 2010). Nevertheless, the observations of expected contrasts, together with the overall agreement between our average white and grey matter $\mu K$ estimates and those obtained by Dhital et al., 2018, strongly support the feasibility of resolving the kurtosis sources according to the CTI methodology in clinical systems.

Disentangling diffusional kurtosis sources without a priori assumptions on the diffusion mode in the tissue represents a new advancement from two different perspectives. On the one hand, it represents an opportunity to enhance specificity in microstructural imaging: the conflation of mesoscopic and microscopic effects in conventional DKI metrics renders the method highly unspecific; separating the sources using CTI thus provides a way to bypass this limitation and quantify the contribution of different sources to the total kurtosis. This could facilitate the detection of different biological processes that may not be visible in conventional DKI due to their counterbalancing effects on overall tissue heterogeneity (e.g. decreased fiber dispersion and fiber anisotropy due to fiber degradation leading to decreased $K_{\text {aniso, }}$ and concomitant variations in the extracellular matrix or the intracellular space due to vasogenic or cytotoxic phenomena leading to increased $K_{\text {iso }}$ and variations in $\mu K$ ). Thus, the possibility to separately map each kurtosis source could reveal the relative "weights" contributing to overall tissue heterogeneity. In turn, this may enhance the biological interpretation of the underlying pathophysiology, as already observed for predicting tumor histology (Szczepankiewicz et al., 2015; Szczepankiewicz et al., 2016; Nilsson et al., 2020) and investigating the mechanisms of the early tissue responses to ischemia (Alves et al., 2021). On the other hand, from the perspective of the biophysical modeling of the dMRI signal, signal representations could play a crucial role for the development of dMRI microstructural models: by providing an unbiased picture of signal features, indeed signal representations may help to set the ground for establishing adequate priors to then be adopted in the modeling process, with the aim of further determining valid links between the detected signal features and their biological underpinnings (Novikov et al., 2018). Moreover, the increasingly available "orthogonal" diffusion-based contrasts, such as MDE preparations, together with the exploration of additional dimensions as the diffusion time, have indeed already helped to identify biases, suggest priors, and constructively shape the ranges of validity of different representations and models (e.g. De Santis et al., 2016; Lampinen et al, 2017; Jespersen et al., 2018; Henriques et al., 2019; Lampinen et al., 2019; Henriques et al., 2021c), thus yielding insightful information in the 
progress toward the validation and development of such approaches (Alexander et al., 2017; Dyrby et al., 2018; Jespersen, 2018).

589

590

591

592

593

594

595

596

597

598

599

600

601

602

603

604

605

606

607

608

609

610

611

612

613

614

615

616

\section{Comparison of CTI and MGC-driven approaches}

Several studies have highlighted the limitations and the ambiguity of the SDE PGSE signal in resolving the kurtosis sources (e.g. Henriques et al., 2019), and until very recently MDE approaches relying on the MGC assumption represented the only option to separate the kurtosis sources, implicitly assuming $\mu K=0$. Using the CTI methodology, our study shows clear evidence for residual non-Gaussian diffusion in the healthy adult human brain (the $\mu K$ component, previously referred to as intra-compartmental kurtosis in Henriques et al., 2020), both at the individual level and at the MNI space analysis. The vanishing of $\mu K$ in CSF lends further credence to our measurements, and suggests it cannot be ignored in the human brain, even when using a $3 \mathrm{~T}$ clinical MRI setting. In the brain tissue, the quantitative analysis of the relative weight of the $\mu K$ component on the total kurtosis (in the MNI space analysis) suggests that $\mu K$ accounts for between 8 and $20 \%$ of total kurtosis. In other words, at the length scales probed by the diffusion times achievable in clinical systems (in our system $G_{\max }=80 \mathrm{mT} / \mathrm{m}$ ), non-Gaussian diffusion components might represent up to $20 \%$ of the total Kurtosis. Systems with higher performance gradients, capable to achieve similar diffusion weightings in shorter diffusion time, might potentially reveal even larger weights of the $\mu K$ component, as observed e.g. in Henriques et al., 2021c, in the preclinical setting. Nevertheless, in the current work by assessing the impact of neglecting $\mu K$ in the estimation of $K_{\text {aniso }}$ and $K_{\text {iso }}$, i.e. under the MGC assumption, consistently with the findings in the rat brains the preclinical setting in Henriques et al., 2021c, our data show lower estimates for $K_{\mathrm{t}}$ and larger estimates for $K_{\text {aniso }}$ and $K_{\text {iso }}$ under the MGC assumption, with larger biases for larger (neglected) $\mu K$ values. As highlighted by the results in Jespersen et al., 2019, it is imperative to carefully assess the effects of nonGaussian diffusion on the MGC assumption. In addition, the $\mu K$ may become much more dominant upon disease (c.f. Alves et al., 2021). We believe that the results presented in the current work are crucial for suggesting an initial range of potential bias associated with the MGC assumption in the healthy human brain at the diffusion times adopted in this study, and thus to help the interpretation of $K_{\text {aniso }}$ and $K_{\text {iso }}$ estimates derived under frameworks assuming Gaussian diffusion over different tissue domains. 
618 Importantly, mapping the $\mu K$ component, in addition to informing the frameworks neglecting 619 non-Gaussian diffusion, represents a new source of contrast per se. By definition, indeed, the $620 \mu K$ component represents the residual non-Gaussianity not captured by $K_{\text {aniso }}$ and $K_{\text {iso, }}$, which our current results suggest to be ubiquitous in the human cerebral tissue. In addition to studies directly investigating $\mu K$, insights on residual non-Gaussian diffusion mainly come from the works investigating diffusion and in turn kurtosis time-dependence. Differently from the CTI approach, these require multiple measurements at different diffusion times, which can be onerous for clinical scanning. The observation of time-dependent diffusion, and in turn of kurtosis, coefficients indeed suggests that at least one tissue compartment exhibits nonGaussian diffusion (Fieremans et al., 2016; Novikov et al., 2019; Lee et al., 2020a). Such deviations from Gaussianity are believed to be associated with structural disorder and crosssectional variance characterizing the sampled tissue (Novikov et al., 2014, see also below), and to be modulated by exchange effects (Nilsson et al., 2013 - both intra-cellular and intraextracellular exchange, with the latter potentially dominating at the diffusion times adopted in the current studies, Ianuş et al., 2021). It is important to point out that since the length scale of the displacements sensed by the diffusion encoding depends on the diffusion time $(\Delta)$ adopted (in addition to the substrate diffusion coefficient), at very long diffusion times the $\mu K$ component might vanish due to the substrate's complete coarse-graining. In this situation, diffusion in each non-exchanging compartment then averages out and may be described as a Gaussian, and thus by a uniform effective diffusion coefficient (for a description of the diffusion phenomenon as a coarse-graining process see Novikov et al., 2019). Conversely, the $\mu K$ component is expected to be increasingly important at short diffusion times, a regime at which more pronounced time-dependency has been observed (e.g. Fieremans et al., 2016; Jespersen et al., 2018). This short diffusion time regime is more easily achieved by highperformance gradients, such as those available in preclinical and dedicated human MRI systems (McNab et al., 2013b; Jones et al., 2018; Fan et al., 2020; Lee et al., 2020b; Henriques et al., 2021c; Huang et al., 2021).

Several pieces of evidence both from preclinical and clinical applications suggest the diagnostic potential of getting insights in such non-Gaussian diffusion arising from restrictions or structural disorder. For instance, in the preclinical setting, mapping the $\mu K$ component in the rat brain post-ischemia revealed enhanced sensitivity to stroke regions and allowed more 
650

651

652

653

654

655

656

657

658

659

660

661

662

663

664

665

666

667

668

669

670

In clinical systems, insights into such non-Gaussian diffusion in the human brain so far mainly come from studies investigating diffusion and kurtosis time-dependency. While several studies did not observe time-dependent diffusion effects in the in vivo human brain (Clark et al., 2001; Nilsson et al., 2009), other studies reported diffusion time-dependency both for white matter (Horsfield et al., 1994; Baron and Beaulieu, 2014; Van et al., 2014; Fieremans et al., 2016; Lee et al., 2018; Lee et al., 2020b; Arbabi et al., 2020) and grey matter (Baron and Beaulieu, 2014; Lee et al., 2020a). Transverse to axonal bundles, time-dependent diffusion has been observed to report on two-dimensional structural disorder, in turn associated to the extra-axonal space packing geometry (Burcaw et al., 2015; Fieremans et al., 2016; Lee et al., 2018) and to be influenced by variations in axonal caliber, also referred to as axonal beading or varicosities (Ginsburger et al., 2018). Along axonal fibers, a stronger diffusion time- (or frequency-, for OGSE sequences) dependency has been observed to follow the power-law proposed in Novikov et al., 2014 for short-range disorder (Fieremans et al., 2016; Jespersen et al., 2018; Arbabi et al., 2020; Lee et al., 2020b). A link between the observed time-dependent signal modulations and their biological underpinnings comes from simulations of diffusion in threedimensional reconstructions of histology-derived axonal segments, which clarified that the observed power-law time-dependency along axons arises in association with axonal varicosities (Lee et al., 2020b). Observations for a one-dimensional structural disorder powerlaw time dependency in grey matter, furthermore, suggest that this might be a universal property of the neural tissue (Does et al., 2003; Novikov et al., 2014; Lee et al., 2020a; Lee et al., 2020b).

\section{Initial steps toward clinical translation}

672 Data acquisition for this work required almost one hour per participant: two repetitions of the

673 DDE sets required by the CTI methodology were collected, which combined allowed a 674 reduction of effects resulting from cross-terms with imaging gradients. However, in the group 675 template kurtosis maps we found that the distributions of values derived from opposite- or 676 single-polarities were not qualitatively different. This suggests that imaging cross-terms effects 677 may be weak, thus allowing to reduce the acquisition time by half. The resulting $\sim 30$ minutes 678 long acquisition with a single polarity would still be impractical for routine clinical 679 applications, but further work could decrease the number of directions per DDE set to 680 ultimately reduce the scan time further. Nevertheless, we stress that, as pointed out in 
681 Henriques et al., 2021c, mapping the $\mu K$ component does not require the acquisition of all the

682

683

684

685

686

687

688

689

690

691

692

693

694

695

696

697

698

699

700

701

702

703

704

705

706

707

708

709

710

711

DDE sets required by the CTI methodology: a "direct" estimation of the $\mu K$ component indeed can be accessed via eq. 3, which requires the acquisition of just two DDE sets out of the four required by the complete CTI methodology, together with data for estimating $D_{\mathrm{t}}$. A single repetition of such an acquisition in our protocol would require $\sim 15$ minutes, which despite being a long scan time for some clinical applications, is closer to an acceptable acquisition time. Anyways, future work should further address the test-retest reliability, suggest normative values at specific diffusion times on a larger sample, and investigate the sensitivity and specificity of the $\mu K$ component in physiological (e.g. aging) and disease processes.

\section{Limitations}

This work has some limitations. For our phantom validation, we adopted an isotropic homogeneous phantom characterized by Gaussian diffusion. While our results confirm the expected Gaussian-only diffusion both in the phantom and in the lateral ventricles in the brain, the development of realistic phantoms with different levels of disordered structures mimicking the salient microstructural features believed to be associated with the $\mu K$ component would be beneficial and support the current findings. The development of phantoms is an active and crucial branch in the field of the study of microstructure with dMRI (e.g. Shemesh et al., 2010; Shemesh et al., 2012a; Nilsson et al., 2017; Fieremans and Lee, 2018; Giménez et al., 2018). Several studies have used numerical phantoms to investigate dMRI signal modulations associated with non-Gaussianity arising from restrictions or structural disorder (Ginsburger et al., 2018; Palombo et al., 2018; Henriques et al., 2020; Lee et al., 2020b; Lee et al., 2020c; Henriques et al., 2021c; Alves et al., 2021). However, increasing evidence for the accessibility to these microscopic disorder features via dMRI should prompt new crucial advancements in the manufacturing of physical phantoms allowing a further validation of the multiple kurtosis sources.

In addition, when performing the demonstrative MNI-space single-polarity CTI analysis, data were preprocessed considering both polarities. Future studies should investigate further the impact of reducing the number of directions on the CTI metrics estimation by considering accordingly the subset of data in the whole preprocessing stream.

Another limitation is that the current framework assumes negligible exchange between tissue microdomains. More specifically, it should be noted that the microscopic kurtosis is 
currently estimated as the subtraction of the kurtosis arising from isotropic and anisotropic variances from the total kurtosis, leaving thus the possibility of exchange as a potential contributor. In other words, when two components (even if gaussian) are exchanging in the time-scale of the diffusion experiment (diffusion time, mixing time in the case of CTI), then the total kurtosis and the Z-tensor will exhibit different terms associated with the exchange rates. On subtraction, the difference between these terms can generate a finite exchange-driven microscopic kurtosis. Thus, in principle, the microscopic kurtosis contrast could reflect kurtosis arising from cross sectional variance (positive $\mu K$ ), exchange, or a combination of both. Future studies should thus be designed to further disentangle these two effects and investigate more deeply the biological underpinnings of $\mu K$ in the human brain. Nevertheless, separating the kurtosis arising from microscopic sources and variance in tensor magnitude and anisotropy is expected to be highly useful even before all the specific underpinnings are fully resolved (c.f. the sensitivity of microscopic kurtosis contrast in stroke).

\section{Conclusion}

726 This work demonstrates the translation of the CTI methodology from the preclinical to the clinical MRI setting, prompted by the increasing evidence suggesting the relevance of nonGaussian diffusion in the characterization of the human brain microstructure. While nonGaussian effects have been typically investigated by varying diffusion times, making such acquisitions potentially long and impractical, the CTI methodology offers a more practical alternative which has the potential to be further optimized for clinical applications. Mapping the microscopic kurtosis in human brain tissue for the first time revealed that, while until now commonly neglected, this component is non-vanishing. Consistent with this, we show that ignoring microscopic kurtosis affects the estimation of the other kurtosis components. The possibility of mapping microscopic kurtosis in humans opens an intriguing new window on microscopic tissue features of great clinical and neuroscientific interest.

\section{Acknowledgements}

738 The authors thank Stefano Tambalo for support with data acquisition. This research was supported by the Caritro Foundation, Italy, the European Research Council (ERC) under the

740 European Union's Horizon 2020 research and innovation programme (Starting Grant, 741 agreement No. 679058), "la Caixa" Foundation (ID 100010434), and European Union's 
Horizon 2020 research and innovation programme under the Marie Skłodowska-Curie grant agreement No 847648, fellowship code CF/BQ/PI20/11760029.

\section{References}

Aggarwal, M., Jones, M. V., Calabresi, P.A., Mori, S., Zhang, J., 2012. Probing mouse brain microstructure using oscillating gradient diffusion MRI. Magn. Reson. Med. 67, 98-109. https://doi.org/10.1002/mrm.22981

Alexander, D.C., Dyrby, T.B., Nilsson, M., Zhang, H., 2017. Imaging brain microstructure with diffusion MRI: practicality and applications. NMR Biomed. 126. https://doi.org/10.1002/nbm.3841

Alves, R., Henriques, R.N., Kerkelä, L., Chavarrías, C., Jespersen, S.N., Shemesh, N., 2021. Unraveling micro-architectural modulations in neural tissue upon ischemia by Correlation Tensor MRI. bioRxiv 2021.02.20.432088.

Andersen, K.W., Lasič, S., Lundell, H., Nilsson, M., Topgaard, D., Sellebjerg, F., Szczepankiewicz, F., Siebner, H.R., Blinkenberg, M., Dyrby, T.B., 2020. Disentangling white-matter damage from physiological fibre orientation dispersion in multiple sclerosis. Brain Commun. 2. https://doi.org/10.1093/braincomms/fcaa077

Andersson, J.L.R., Skare, S., Ashburner, J., 2003. How to correct susceptibility distortions in spin-echo echo-planar images: application to diffusion tensor imaging. Neuroimage 20, 870-888. https://doi.org/10.1016/S1053-8119(03)003367

Andersson, J.L.R., Sotiropoulos, S.N., 2016. An integrated approach to correction for off-resonance effects and subject movement in diffusion MR imaging. Neuroimage 125, 1063-1078. https://doi.org/10.1016/j.neuroimage.2015.10.019

Anwander, A., Tittgemeyer, M., Von Cramon, D.Y., Friederici, A.D., Knösche, T.R., 2007. Connectivity-based parcellation of Broca's area. Cereb. Cortex 17, 816-825. https://doi.org/10.1093/cercor/bhk034

Arab, A., Wojna-Pelczar, A., Khairnar, A., Szabó, N., Ruda-Kucerova, J., 2018. Principles of diffusion kurtosis imaging and its role in early diagnosis of neurodegenerative disorders. Brain Res. Bull. 139, 91-98. https://doi.org/10.1016/J.BRAINRESBULL.2018.01.015

Arbabi, A., Kai, J., Khan, A.R., Baron, C.A., 2020. Diffusion dispersion imaging: Mapping oscillating gradient spin-echo frequency dependence in the human brain. Magn. Reson. Med. 83, 2197-2208. https://doi.org/10.1002/mrm.28083 
Assaf, Y., Cohen, Y., 1998. Non-Mono-Exponential Attenuation of Water and N-Acetyl Aspartate Signals Due to Diffusion in Brain Tissue. J. Magn. Reson. 131, 69-85. https://doi.org/10.1006/jmre.1997.1313

Assaf, Y., Pasternak, O., 2008. Diffusion tensor imaging (DTI)-based white matter mapping in brain research: A review. J. Mol. Neurosci. 34, 51-61. https://doi.org/10.1007/s12031-007-0029-0

Baron, C.A., Beaulieu, C., 2014. Oscillating gradient spin-echo (OGSE) diffusion tensor imaging of the human brain. Magn. Reson. Med. 72, 726-736. https://doi.org/10.1002/mrm.24987

Baron, C.A. lla., Kate, M., Gioia, L., Butcher, K., Emery, D., Budde, M., Beaulieu, C., 2015. Reduction of Diffusion-Weighted Imaging Contrast of Acute Ischemic Stroke at Short Diffusion Times. Stroke. 46, 2136-2141. https://doi.org/10.1161/STROKEAHA.115.008815

Basser, P.J., Mattiello, J., LeBihan, D., 1994. MR diffusion tensor spectroscopy and imaging. Biophys. J. 66, 259-267. https://doi.org/10.1016/S0006-3495(94)80775-1

Bastiani, M., Cottaar, M., Fitzgibbon, S.P., Suri, S., Alfaro-Almagro, F., Sotiropoulos, S.N., Jbabdi, S., Andersson, J.L.R., 2019. Automated quality control for within and between studies diffusion MRI data using a non-parametric framework for movement and distortion correction. Neuroimage 184, 801-812. https://doi.org/10.1016/j.neuroimage.2018.09.073

Benetti, S., Novello, L., Maffei, C., Rabini, G., Jovicich, J., Collignon, O., 2018. White matter connectivity between occipital and temporal regions involved in face and voice processing in hearing and early deaf individuals. Neuroimage 179, 263-274. https://doi.org/10.1016/j.neuroimage.2018.06.044

Blumenfeld-Katzir, T., Pasternak, O., Dagan, M., Assaf, Y., 2011. Diffusion MRI of structural brain plasticity induced by a learning and memory task. PLoS One 6. https://doi.org/10.1371/journal.pone.0020678

Budde, M.D., Skinner, N.P., Tugan Muftuler, L., Schmit, B.D., Kurpad, S.N., 2017. Optimizing filter-probe diffusion weighting in the rat spinal cord for human translation. Front. Neurosci. 11, 1-13. https://doi.org/10.3389/fnins.2017.00706

Burcaw, L.M., Fieremans, E., Novikov, D.S., 2015. Mesoscopic structure of neuronal tracts from time-dependent diffusion. Neuroimage 114, 18-37. https://doi.org/10.1016/j.neuroimage.2015.03.061

Callaghan, P., Coy, A., MacGowan, D., Packer K.J., Zelaya F.O., 1991. Diffraction-like effects in NMR diffusion studies of fluids in porous solids. Nature 351, 467-469. https://doi.org/10.1038/351467a0 
Callaghan, P.T., Komlosh, M.E., 2002. Locally anisotropic motion in a macroscopically isotropic system: Displacement correlations measured using double pulsed gradient spin-echo NMR. Magn. Reson. Chem. 40, 15-19. https://doi.org/10.1002/mrc.1122

Cheng, Y., Cory, D.G., 1999. Multiple scattering by NMR [3]. J. Am. Chem. Soc. 121, 7935-7936. https://doi.org/10.1021/ja9843324

Clark, C.A., Hedehus, M., Moseley, M.E., 2001. Diffusion time dependence of the apparent diffusion tensor in healthy human brain and white matter disease. Magn. Reson. Med. 45, 1126-1129. https://doi.org/10.1002/mrm.1149

De Santis, S., Jones, D.K., Roebroeck, A., 2016. Including diffusion time dependence in the extra-axonal space improves in vivo estimates of axonal diameter and density in human white matter. Neuroimage 130, 91-103. https://doi.org/10.1016/j.neuroimage.2016.01.047

Delgado, A.F., Nilsson, M., Van Westen, D., Delgado, A.F., 2018. Glioma grade discrimination with MR diffusion kurtosis imaging: A meta-Analysis of diagnostic accuracy. Radiology 287, 119-127. https://doi.org/10.1148/radiol.2017171315

Della-Maggiore, V., Scholz, J., Johansen-Berg, H., Paus, T., 2009. The rate of visuomotor adaptation correlates with cerebellar white-matter microstructure. Hum. Brain Mapp. 30, 4048-4053. https://doi.org/10.1002/hbm.20828

Dhital, B., Kellner, E., Kiselev, V.G., Reisert, M., 2018. The absence of restricted water pool in brain white matter. Neuroimage 182, 398-406. https://doi.org/10.1016/j.neuroimage.2017.10.051

Does, M.D., Parsons, E.C., Gore, J.C., 2003. Oscillating gradient measurements of water diffusion in normal and globally ischemic rat brain. Magn. Reson. Med. 49, 206215. https://doi.org/10.1002/mrm.10385

Dyrby, T.B., Innocenti, G., Bech, M., Lundell, H., 2018. Validation strategies for the interpretation of microstructure imaging using diffusion MRI. Neuroimage. https://doi.org/10.1016/j.neuroimage.2018.06.049

Eriksson, S., Lasic, S., Topgaard, D., 2013. Isotropic diffusion weighting in PGSE NMR by magic-angle spinning of the q-vector. J. Magn. Reson. 226, 13-18. https://doi.org/10.1016/J.JMR.2012.10.015

Falangola, M.F., Jensen, J.H., Babb, J.S., Hu, C., Castellanos, F.X., Di Martino, A., Ferris, S.H., Helpern, J.A., 2008. Age-related non-Gaussian diffusion patterns in the prefrontal brain. J. Magn. Reson. Imaging 28, 1345-1350. https://doi.org/10.1002/jmri.21604

Fan, Q., Witzel, T., Tounekti, S., Tian, Q., Ngamsombat, C., Polackal, M., 

different cortical regions using double-diffusion encoding, in: Proc. Intl. Soc. Mag. Reson. Med.

Fieremans, E., Benitez, A., Jensen, J.H., Falangola, M.F., Tabesh, A., Deardorff, R.L., Novel white matter tract integrity metrics sensitive to Alzheimer disease progression. Am. J. Neuroradiol. 34, 2105-2112. https://doi.org/10.3174/ajnr.A3553

855

856

857

858

859

860

861

862

863

864

865

866

867

868

869

870

871

872

873

874

875

876

877

878

879

880

881

882

883

Fieremans, E., Burcaw, L.M., Lee, H.H., Lemberskiy, G., Veraart, J., Novikov, D.S., 2016. In vivo observation and biophysical interpretation of time-dependent diffusion in human white matter. Neuroimage 129, 414-427. https://doi.org/10.1016/j.neuroimage.2016.01.018

Fieremans, E., Jensen, J.H., Helpern, J.A., 2011. White matter characterization with diffusional kurtosis imaging. Neuroimage 58, 177-188. https://doi.org/10.1016/j.neuroimage.2011.06.006

Fieremans, E., Lee, H.H., 2018. Physical and numerical phantoms for the validation of brain microstructural MRI: A cookbook. Neuroimage. https://doi.org/10.1016/j.neuroimage.2018.06.046

Gauvain, K.M., McKinstry, R.C., Mukherjee, P., Perry, A., Neil, J.J., Kaufman, B.A., Hayashi, R.J., 2001. Evaluating pediatric brain tumor cellularity with diffusiontensor imaging. Am. J. Roentgenol. 177, 449-454. https://doi.org/10.2214/ajr.177.2.1770449

Giménez, M.L., Jiménez, P., Pedraza Pérez, L.A., Betancourth, D., Zwick, A., Álvarez, G.A., 2018. Microstructure size-distribution estimations with smooth and sharp non-uniform oscillating gradient spin-echo modulations, in: Proc Intl Soc Mag Reson Med.

Ginsburger, K., Poupon, F., Beaujoin, J., Estournet, D., Matuschke, F., Mangin, J.F., Axer, M., Poupon, C., 2018. Improving the realism of white matter numerical phantoms: A step toward a better understanding of the influence of structural disorders in diffusion MRI. Front. Phys. 5. https://doi.org/10.3389/fphy.2018.00012

Gong, N.J., Wong, C.S., Chan, C.C., Leung, L.M., Chu, Y.C., 2013. Correlations between microstructural alterations and severity of cognitive deficiency in Alzheimer's disease and mild cognitive impairment: a diffusional kurtosis imaging study. Magn. Reson. Imaging 31, 688-694. https://doi.org/10.1016/J.MRI.2012.10.027

Grussu, F., Ianuş, A., Tur, C., Prados, F., Schneider, T., Kaden, E., Ourselin, S., Drobnjak, I., Zhang, H., Alexander, D.C., Gandini Wheeler-Kingshott, C.A.M., 
2019. Relevance of time-dependence for clinically viable diffusion imaging of the spinal cord. Magn. Reson. Med. 81, 1247-1264. https://doi.org/10.1002/mrm.27463

Hardin RH, Sloane NJAA. McLaren's improved snub cube and other new spherical designs in three dimensions. Discret Comput Geom. 1996;15:429- 441

Hasan, K.M., Mwangi, B., Keser, Z., Riascos, R., Sargsyan, A.E., Kramer, L.A., 2018. Brain Quantitative MRI Metrics in Astronauts as a Unique Professional Group. J. Neuroimaging 28, 256-268. https://doi.org/10.1111/jon.12501

Helpern, J.A., Adisetiyo, V., Falangola, M.F., Hu, C., Di Martino, A., Williams, K., Castellanos, F.X., Jensen, J.H., 2011. Preliminary evidence of altered gray and white matter microstructural development in the frontal lobe of adolescents with attention-deficit hyperactivity disorder: A diffusional kurtosis imaging study. J. Magn. Reson. Imaging 33, 17-23. https://doi.org/10.1002/jmri.22397

Hempel, J.M., Bisdas, S., Schittenhelm, J., Brendle, C., Bender, B., Wassmann, H., Skardelly, M., Tabatabai, G., Vega, S.C., Ernemann, U., Klose, U., 2017. In vivo molecular profiling of human glioma using diffusion kurtosis imaging. J. Neurooncol. 131, 93-101. https://doi.org/10.1007/s11060-016-2272-0

Hempel, J.M., Schittenhelm, J., Bisdas, S., Brendle, C., Bender, B., Bier, G., Skardelly, M., Tabatabai, G., Castaneda Vega, S., Ernemann, U., Klose, U., 2018. In vivo assessment of tumor heterogeneity in WHO 2016 glioma grades using diffusion kurtosis imaging: Diagnostic performance and improvement of feasibility in routine clinical practice. J. Neuroradiol. 45, 32-40. https://doi.org/10.1016/J.NEURAD.2017.07.005

Henriques, R.N., 2018. Advanced Methods for Diffusion MRI Data Analysis and their Application to the Healthy Ageing Brain. University of Cambridge. https://doi.org/https://doi.org/10.17863/CAM.29356

Henriques, R.N., Correia, M.M., Marrale, M., Huber, E., Kruper, J., Koudoro, S., Yeatman, J.D., Garyfallidis, E., Rokem, A., 2021a. Diffusional Kurtosis Imaging in the Diffusion Imaging in Python Project. Front. Hum. Neurosci. 15. https://doi.org/10.3389/fnhum.2021.675433

Henriques, R.N., Correia, M.M., Nunes, R.G., Ferreira, H.A., 2015. Exploring the 3D geometry of the diffusion kurtosis tensor-Impact on the development of robust tractography procedures and novel biomarkers. Neuroimage 111, 85-99. https://doi.org/10.1016/J.NEUROIMAGE.2015.02.004

Henriques, R.N., Jespersen, S.N., Jones, D.K., Veraart, J., 2021d. Toward more robust and reproducible diffusion kurtosis imaging. Magn. Reson. Med. 86, 1600-1613. https://doi.org/10.1002/mrm.28730 
936

Henriques, R.N., Jespersen, S.N., Shemesh, N., 2020. Correlation tensor magnetic resonance imaging. Neuroimage 211. https://doi.org/10.1016/j.neuroimage.2020.116605

Henriques, R.N., Jespersen, S.N., Shemesh, N., 2019. Microscopic anisotropy misestimation in spherical-mean single diffusion encoding MRI. Magn. Reson. Med. 81, 3245-3261. https://doi.org/10.1002/mrm.27606

Henriques, R.N., Palombo, M., Jespersen, S.N., Shemesh, N., Lundell, H., Ianuş, A., 2021b. Double diffusion encoding and applications for biomedical imaging. J. Neurosci. Methods 108989. https://doi.org/10.1016/j.jneumeth.2020.108989

Henriques, R.N., Jespersen, S.N., Shemesh, N., 2021c. Evidence for microscopic kurtosis in neural tissue revealed by correlation tensor MRI. Magn. Reson. Med. 120. https://doi.org/10.1002/mrm.28938

Horsfield, M.A., Barker, G.J., McDonald, W.I., 1994. Self-diffusion in CNS tissue by volume-selective proton NMR. Magn. Reson. Med. 31, 637-644. https://doi.org/10.1002/mrm.1910310609

Huang, S.Y., Witzel, T., Keil, B., Scholz, A., Davids, M., Dietz, P., Rummert, E., Ramb, R., Kirsch, J.E., Yendiki, A., Fan, Q., Tian, Q., Ramos-Llordén, G., Lee, H.H., Nummenmaa, A., Bilgic, B., Setsompop, K., Wang, F., Avram, A. V., Komlosh, M., Benjamini, D., Magdoom, K.N., Pathak, S., Schneider, W., Novikov, D.S., Fieremans, E., Tounekti, S., Mekkaoui, C., Augustinack, J., Berger, D., ShapsonCoe, A., Lichtman, J., Basser, P.J., Wald, L.L., Rosen, B.R., 2021. Connectome 2.0: Developing the next-generation ultra-high gradient strength human MRI scanner for bridging studies of the micro-, meso- and macro-connectome. Neuroimage 243, 118530. https://doi.org/10.1016/j.neuroimage.2021.118530

Huber, E., Henriques, R.N., Owen, J.P., Rokem, A., Yeatman, J.D., 2019. Applying microstructural models to understand the role of white matter in cognitive development. Dev. Cogn. Neurosci. 36, 100624. https://doi.org/10.1016/J.DCN.2019.100624

Hui, E.S., Fieremans, E., Jensen, J.H., Tabesh, A., Feng, W., Bonilha, L., Spampinato, M. V., Adams, R., Helpern, J.A., 2012. Stroke assessment with diffusional kurtosis imaging. Stroke 43, 2968-2973. https://doi.org/10.1161/STROKEAHA.112.657742

Ianus, A., Alexander, D.C., Zhang, H., Palombo, M., 2021. Mapping complex cell morphology in the grey matter with double diffusion encoding MR: A simulation study. Neuroimage 241, 118424. https://doi.org/10.1016/J.NEUROIMAGE.2021.118424 
Ianuş, A., Drobnjak, I., Alexander, D.C., 2016. Model-based estimation of microscopic anisotropy using diffusion MRI: A simulation study. NMR Biomed. 29, 672-685. https://doi.org/10.1002/nbm.3496

Ianuş, A., Jespersen, S.N., Serradas Duarte, T., Alexander, D.C., Drobnjak, I., Shemesh, N., 2018. Accurate estimation of microscopic diffusion anisotropy and its time dependence in the mouse brain. Neuroimage 183, 934-949. https://doi.org/10.1016/j.neuroimage.2018.08.034

Ianuş, A., Shemesh, N., Alexander, D.C., Drobnjak, I., 2017. Double Oscillating Diffusion Encoding and Sensitivity to Microscopic Anisotropy. Magn. Reson. Med. 78, 550-564. https://doi.org/10.1002/mrm.26393

Jacobacci, F., Armony, J.L., Yeffal, A., Lerner, G., Amaro, E., Jovicich, J., Doyone, J., Della-Maggiore, V., 2020. Rapid hippocampal plasticity supports motor sequence learning. Proc. Natl. Acad. Sci. U. S. A. 117, 23898-23903. https://doi.org/10.1073/pnas.2009576117

Jelescu, I.O., Veraart, J., Adisetiyo, V., Milla, S.S., Novikov, D.S., Fieremans, E., 2015. One diffusion acquisition and different white matter models: How does microstructure change in human early development based on WMTI and NODDI? Neuroimage 107, 242-256. https://doi.org/10.1016/J.NEUROIMAGE.2014.12.009

Jelescu, I.O., Veraart, J., Fieremans, E., Novikov, D.S., 2016. Degeneracy in model parameter estimation for multi-compartmental diffusion in neuronal tissue. NMR Biomed. 29, 33-47. https://doi.org/10.1002/nbm.3450

Jensen, J.H., Helpern, J.A., 2010. MRI quantification of non-Gaussian water diffusion by kurtosis analysis. NMR Biomed. https://doi.org/10.1002/nbm.1518

Jensen, J.H., Helpern, J.A., Ramani, A., Lu, H., Kaczynski, K., 2005. Diffusional kurtosis imaging: The quantification of non-Gaussian water diffusion by means of magnetic resonance imaging. Magn. Reson. Med. 53, 1432-1440. https://doi.org/10.1002/mrm.20508

Jespersen, S.N., Kroenke, C.D., Østergaard, L., Ackerman, J.J.H., Yablonskiy, D.A., 2007. Modeling dendrite density from magnetic resonance diffusion measurements. Neuroimage 34, 1473-1486. https://doi.org/10.1016/j.neuroimage.2006.10.037

Jespersen, S.N., 2018. White matter biomarkers from diffusion MRI. J. Magn. Reson. 291, 127-140. https://doi.org/10.1016/J.JMR.2018.03.001

Jespersen, S.N., 2012. Equivalence of double and single wave vector diffusion contrast at low diffusion weighting. NMR Biomed. 25, 813-818. https://doi.org/10.1002/nbm.1808 
Jespersen, S.N., Buhl, N., 2011. The displacement correlation tensor: Microstructure, ensemble anisotropy and curving fibers. J. Magn. Reson. 208, 34-43. https://doi.org/10.1016/j.jmr.2010.10.003

Jespersen, S.N., Lundell, H., Sønderby, C.K., Dyrby, T.B., 2013. Orientationally invariant metrics of apparent compartment eccentricity from double pulsed field gradient diffusion experiments. NMR Biomed. 26, 1647-1662. https://doi.org/10.1002/nbm.2999

Jespersen, S.N., Olesen, J.L., Hansen, B., Shemesh, N., 2018. Diffusion time dependence of microstructural parameters in fixed spinal cord. Neuroimage 182, 329-342. https://doi.org/10.1016/j.neuroimage.2017.08.039

Jespersen, S.N., Olesen, J.L., Ianuş, A., Shemesh, N., 2019. Effects of nongaussian diffusion on "isotropic diffusion" measurements: An ex-vivo microimaging and simulation study. J. Magn. Reson. 300, 84-94. https://doi.org/10.1016/J.JMR.2019.01.007

Johansen-Berg, H., 2010. Behavioural relevance of variation in white matter microstructure. Curr. Opin. Neurol. 23, 351-358. https://doi.org/10.1097/WCO.0b013e32833b7631

Jones, D.K., Alexander, D.C., Bowtell, R., Cercignani, M., Dell'Acqua, F., McHugh, D.J., Miller, K.L., Palombo, M., Parker, G.J.M., Rudrapatna, U.S., Tax, C.M.W., 2018. Microstructural imaging of the human brain with a 'super-scanner': 10 key advantages of ultra-strong gradients for diffusion MRI. Neuroimage 182, 8-38. https://doi.org/10.1016/j.neuroimage.2018.05.047

Kaden, E., Kelm, N.D., Carson, R.P., Does, M.D., Alexander, D.C., 2016. Multicompartment microscopic diffusion imaging. Neuroimage 139, 346-359. https://doi.org/10.1016/j.neuroimage.2016.06.002

Kamagata, K., Tomiyama, H., Hatano, T., Motoi, Y., Abe, O., Shimoji, K., Kamiya, K., Suzuki, M., Hori, M., Yoshida, M., Hattori, N., Aoki, S., 2014. A preliminary diffusional kurtosis imaging study of Parkinson disease: Comparison with conventional diffusion tensor imaging. Neuroradiology 56, 251-258. https://doi.org/10.1007/s00234-014-1327-1

Kamagata, K., Tomiyama, H., Motoi, Y., Kano, M., Abe, O., Ito, K., Shimoji, K., Suzuki, M., Hori, M., Nakanishi, A., Kuwatsuru, R., Sasai, K., Aoki, S., Hattori, N., 2013. Diffusional kurtosis imaging of cingulate fibers in Parkinson disease: Comparison with conventional diffusion tensor imaging. Magn. Reson. Imaging 31, 1501-1506. https://doi.org/10.1016/J.MRI.2013.06.009

Kamiya, K., Kamagata, K., Ogaki, K., Hatano, T., Ogawa, T., Takeshige-Amano, H., Murata, S., Andica, C., Murata, K., Feiweier, T., Hori, M., Hattori, N., Aoki, S., 
2020. Brain White-Matter Degeneration Due to Aging and Parkinson Disease as Revealed by Double Diffusion Encoding. Front. Neurosci. 14, 1-15. https://doi.org/10.3389/fnins.2020.584510

Kellner, E., Dhital, B., Kiselev, V.G., Reisert, M., 2016. Gibbs-ringing artifact removal based on local subvoxel-shifts. Magn. Reson. Med. 76, 1574-1581. https://doi.org/10.1002/mrm.26054

Kerkelä, L., Henriques, R.N., Hall, M.G., Clark, C.A., Shemesh, N., 2019. Validation and noise robustness assessment of microscopic anisotropy estimation with clinically feasible double diffusion encoding MRI. Magn. Reson. Med. https://doi.org/10.1002/mrm.28048

Komlosh, M.E., Horkay, F., Freidlin, R.Z., Nevo, U., Assaf, Y., Basser, P.J., 2007. Detection of microscopic anisotropy in gray matter and in a novel tissue phantom using double Pulsed Gradient Spin Echo MR. J. Magn. Reson. 189, 38-45. https://doi.org/10.1016/J.JMR.2007.07.003

Komlosh, M.E., Lizak, M.J., Horkay, F., Freidlin, R.Z., Basser, P.J., 2008. Observation of microscopic diffusion anisotropy in the spinal cord using double-pulsed gradient spin echo MRI. Magn. Reson. Med. 59, 803-809. https://doi.org/10.1002/mrm.21528

Kunz, N., Sizonenko, S. V., Hüppi, P.S., Gruetter, R., Van de Looij, Y., 2013. Investigation of field and diffusion time dependence of the diffusion-weighted signal at ultrahigh magnetic fields. NMR Biomed. 26, 1251-1257. https://doi.org/10.1002/nbm.2945

Lampinen, B., Szczepankiewicz, F., Mårtensson, J., van Westen, D., Sundgren, P.C., Nilsson, M., 2017. Neurite density imaging versus imaging of microscopic anisotropy in diffusion MRI: A model comparison using spherical tensor encoding. Neuroimage 147, 517-531. https://doi.org/10.1016/j.neuroimage.2016.11.053

Lampinen, B., Szczepankiewicz, F., Novén, M., van Westen, D., Hansson, O., Englund, E., Mårtensson, J., Westin, C.F., Nilsson, M., 2019. Searching for the neurite density with diffusion MRI: Challenges for biophysical modeling. Hum. Brain Mapp. 40, 2529-2545. https://doi.org/10.1002/hbm.24542

Lasič, S., Szczepankiewicz, F., Eriksson, S., Nilsson, M., Topgaard, D., 2014. Microanisotropy imaging: quantification of microscopic diffusion anisotropy and orientation of order parameter by diffusion MRI with magic-angle spinning of the q-vectorâ€. Front. Phys. 2, 1-14. https://doi.org/10.3389/fphy.2014.00028

Lawrenz, M., Brassen, S., Finsterbusch, J., 2016. Microscopic diffusion anisotropy in the human brain: Age-related changes. Neuroimage 141, 313-325. https://doi.org/10.1016/J.NEUROIMAGE.2016.07.031 
Lawrenz, M., Finsterbusch, J., 2019. Detection of microscopic diffusion anisotropy in human cortical gray matter in vivo with double diffusion encoding. Magn. Reson. Med. 81, 1296-1306. https://doi.org/10.1002/mrm.27451

Lawrenz, M., Finsterbusch, J., 2015. Mapping measures of microscopic diffusion anisotropy in human brain white matter in vivo with double-wave-vector diffusionweighted imaging. Magn. Reson. Med. 73, 773-783. https://doi.org/10.1002/mrm.25140

Lawrenz, M., Finsterbusch, J., 2013. Double-Wave-Vector Diffusion-Weighted Imaging Reveals Microscopic Diffusion Anisotropy in the Living Human Brain. Magn. Reson. Med. 1082, 1072-1082. https://doi.org/10.1002/mrm.24347

Lawrenz, M., Finsterbusch, J., 2011. Detection of microscopic diffusion anisotropy on a whole-body MR system with double wave vector imaging. Magn. Reson. Med. 66, 1405-1415. https://doi.org/10.1002/mrm.22934

Lawrenz, M., Koch, M.A., Finsterbusch, J., 2010. A tensor model and measures of microscopic anisotropy for double-wave-vector diffusion-weighting experiments with long mixing times. J. Magn. Reson. 202, 43-56. https://doi.org/10.1016/j.jmr.2009.09.015

Le Bihan, D., Breton, E., Lallemand, D., Grenier, P., Cabanis, E., Laval-Jeantet, M., 1986. MR imaging of intravoxel incoherent motions: application to diffusion and perfusion in neurologic disorders. Radiology 161, 401-407. https://doi.org/10.1148/radiology.161.2.3763909

Lee, H.-H., Jespersen, S.N., Fieremans, E., Novikov, D.S., 2020c. The impact of realistic axonal shape on axon diameter estimation using diffusion MRI. Neuroimage 117228. https://doi.org/10.1016/j.neuroimage.2020.117228

Lee, H.-H., Papaioannou, A., Novikov, D.S., Fieremans, E., 2020a. In vivo observation and biophysical interpretation of time-dependent diffusion in human cortical gray matter. Neuroimage 222, 117054. https://doi.org/10.1016/j.neuroimage.2020.117054

Lee, H.-H., Fieremans, E., Novikov, D.S., 2018. What dominates the time dependence of diffusion transverse to axons: Intra- or extra-axonal water? Neuroimage 182, 500-510. https://doi.org/10.1016/j.neuroimage.2017.12.038

Lee, H.-H., Papaioannou, A., Kim, S.L., Novikov, D.S., Fieremans, E., 2020b. A timedependent diffusion MRI signature of axon caliber variations and beading. Commun. Biol. 3. https://doi.org/10.1038/s42003-020-1050-x 
Leemans, A., Jeurissen, B., Sijbers, J., Jones, D.K., 2009. ExploreDTI: a graphical toolbox for processing, analyzing, and visualizing diffusion MR data. Proc. Intl. Soc. Mag Reson. Med. 17, 3537.

Lin, L., Bhawana, R., Xue, Y., Duan, Q., Jiang, R., Chen, H., Chen, X., Sun, B., Lin, H., 2018. Comparative analysis of diffusional kurtosis imaging, diffusion tensor imaging, and diffusion-weighted imaging in grading and assessing cellular proliferation of meningiomas. Am. J. Neuroradiol. 39, 1032-1038. https://doi.org/10.3174/ajnr.A5662

Lu, H., Jensen, J.H., Ramani, A., Helpern, J.A., 2006. Three-dimensional characterization of non-gaussian water diffusion in humans using diffusion kurtosis imaging. NMR Biomed. 19, 236-247. https://doi.org/10.1002/nbm.1020

McNab, J.A., Polimeni, J.R., Wang, R., Augustinack, J.C., Fujimoto, K., Stevens, A., Janssens, T., Farivar, R., Folkerth, R.D., Vanduffel, W., Wald, L.L., 2013a. Surface based analysis of diffusion orientation for identifying architectonic domains in the in vivo human cortex. Neuroimage 69, 87-100. https://doi.org/10.1016/J.NEUROIMAGE.2012.11.065

McNab, J.A., Edlow, B.L., Witzel, T., Huang, S.Y., Bhat, H., Heberlein, K., Feiweier, T., Liu, K., Keil, B., Cohen-Adad, J., Tisdall, M.D., Folkerth, R.D., Kinney, H.C., Wald, L.L., 2013b. The Human Connectome Project and beyond: Initial applications of $300 \mathrm{mT} / \mathrm{m}$ gradients. Neuroimage $80,234-245$. https://doi.org/10.1016/j.neuroimage.2013.05.074

Mitra, P.P., 1995. Multiple wave-vector extensions of the NMR pulsed-field-gradient spin-echo diffusion measurement. Phys. Rev. B 51, 74-78. https://doi.org/https://journals.aps.org/prb/abstract/10.1103/PhysRevB.51.15074

Mohammadi, S., Tabelow, K., Ruthotto, L., Feiweier, T., Polzehl, J., Weiskopf, N., 2015. High-resolution diffusion kurtosis imaging at 3T enabled by advanced postprocessing. Front. Neurosci. 9, 427. https://doi.org/10.3389/fnins.2014.00427

Moseley, M.E., Cohen, Y., Mintorovitch, J., Chileuitt, L., Shimizu, H., Kucharczyk, J., Wendland, M.F., Weinstein, P.R., 1990. Early detection of regional cerebral ischemia in cats: Comparison of diffusion- and T2-weighted MRI and spectroscopy. Magn. Reson. Med. 14, 330-346. https://doi.org/10.1002/mrm.1910140218

Mueller, L., Wetscherek, A., Kuder, T.A., Laun, F.B., 2017. Eddy current compensated double diffusion encoded (DDE) MRI. Magn. Reson. Med. 77, 328-335. https://doi.org/10.1002/mrm.26092

Mukherjee, P., 2005. Diffusion tensor imaging and fiber tractography in acute stroke. Neuroimaging Clin. N. Am. 15, 655-665. https://doi.org/10.1016/j.nic.2005.08.010 
Mulkern, R. V., Gudbjartsson, H., Westin, C.F., Zengingonul, H.P., Gartner, W., Guttmann, C.R.G., Robertson, R.L., Kyriakos, W., Schwartz, R., Holtzman, D., Jolesz, F.A., Maier, S.E., 1999. Multi-component apparent diffusion coefficients in human brain. NMR Biomed. 12, 51-62. https://doi.org/10.1002/(SICI)10991492(199902)12:1<51::AID-NBM546>3.0.CO;2-E

Neeman, M., Freyer, J.P., Sillerud, L.O., 1991. A simple method for obtaining crossterm-free images for diffusion anisotropy studies in NMR microimaging. Magn. Reson. Med. 21, 138-143. https://doi.org/10.1002/mrm.1910210117

Nilsson, M., Lasič, S., Drobnjak, I., Topgaard, D., Westin, C.F., 2017. Resolution limit of cylinder diameter estimation by diffusion MRI: The impact of gradient waveform and orientation dispersion. NMR Biomed. 30, 1-13. https://doi.org/10.1002/nbm.3711

Nilsson, M., Lätt, J., Nordh, E., Wirestam, R., Ståhlberg, F., Brockstedt, S., 2009. On the effects of a varied diffusion time in vivo: is the diffusion in white matter restricted? Magn. Reson. Imaging 27, 176-187. https://doi.org/10.1016/J.MRI.2008.06.003

Nilsson, M., Lätt, J., Van Westen, D., Brockstedt, S., Lasič, S., Ståhlberg, F., Topgaard, D., 2013. Noninvasive mapping of water diffusional exchange in the human brain using filter-exchange imaging. Magn. Reson. Med. 69, 1572-1580. https://doi.org/10.1002/mrm.24395

Nilsson, M., Szczepankiewicz, F., Brabec, J., Taylor, M., Westin, C.F., Golby, A., van Westen, D., Sundgren, P.C., 2020. Tensor-valued diffusion MRI in under 3 minutes: an initial survey of microscopic anisotropy and tissue heterogeneity in intracranial tumors. Magn. Reson. Med. 83, 608-620. https://doi.org/10.1002/mrm.27959

Novikov, D.S., 2021. The present and the future of microstructure MRI: From a paradigm shift to normal science. J. Neurosci. Methods 351, 108947. https://doi.org/10.1016/j.jneumeth.2020.108947

Novikov, D.S., Fieremans, E., Jespersen, S.N., Kiselev, V.G., 2019. Quantifying brain microstructure with diffusion MRI: Theory and parameter estimation. NMR Biomed. 32, 1-53. https://doi.org/10.1002/nbm.3998

Novikov, D.S., Jensen, J.H., Helpern, J.A., Fieremans, E., 2014. Revealing mesoscopic structural universality with diffusion. Proc. Natl. Acad. Sci. U. S. A. 111, 50885093. https://doi.org/10.1073/pnas.1316944111

Novikov, D.S., Kiselev, V.G., Jespersen, S.N., 2018. On modeling. Magn. Reson. Med. 79, 3172-3193. https://doi.org/10.1002/mrm.27101 
Özarslan, E., 2009. Compartment shape anisotropy (CSA) revealed by double pulsed field gradient MR. J. Magn. Reson. 199, 56-67. https://doi.org/10.1016/J.JMR.2009.04.002

Özarslan, E., Basser, P.J., 2008. Microscopic anisotropy revealed by NMR double pulsed field gradient experiments with arbitrary timing parameters. J. Chem. Phys. 128. https://doi.org/10.1063/1.2905765

Palombo, M., Ligneul, C., Hernandez-Garzon, E., Valette, J., 2018. Can we detect the effect of spines and leaflets on the diffusion of brain intracellular metabolites? Neuroimage 182, 283-293. https://doi.org/10.1016/j.neuroimage.2017.05.003

Paulsen, J.L., Özarslan, E., Komlosh, M.E., Basser, P.J., Song, Y.Q., 2015. Detecting compartmental non-Gaussian diffusion with symmetrized double-PFG MRI. NMR Biomed. 28, 1550-1556. https://doi.org/10.1002/nbm.3363

Portnoy, S., Flint, J.J., Blackband, S.J., Stanisz, G.J., 2013. Oscillating and pulsed gradient diffusion magnetic resonance microscopy over an extended b-value range: Implications for the characterization of tissue microstructure. Magn. Reson. Med. 69, 1131-1145. https://doi.org/10.1002/mrm.24325

Raab, P., Hattingen, E., Franz, K., Zanella, F.E., 2010. Cerebral gliomas: diffusional kurtosis imaging analysis of microstructural differences. Radiology 254.

Raja, R., Sinha, N., Saini, J., Mahadevan, A., Rao, K.N., Swaminathan, A., 2016. Assessment of tissue heterogeneity using diffusion tensor and diffusion kurtosis imaging for grading gliomas. Neuroradiology 58, 1217-1231. https://doi.org/10.1007/s00234-016-1758-y

Roebroeck, A., Galuske, R., Formisano, E., Chiry, O., Bratzke, H., Ronen, I., Kim, D. shik, Goebel, R., 2008. High-resolution diffusion tensor imaging and tractography of the human optic chiasm at $9.4 \mathrm{~T}$. Neuroimage $39,157-168$. https://doi.org/10.1016/j.neuroimage.2007.08.015

Scholz, J., Klein, M.C., Behrens, T.E.J., Johansen-Berg, H., 2009. Training induces changes in white-matter architecture. Nat. Neurosci. 12, 1370-1371. https://doi.org/10.1038/nn.2412

Shemesh, N., 2018. Axon Diameters and Myelin Content Modulate Microscopic Fractional Anisotropy at Short Diffusion Times in Fixed Rat Spinal Cord. Front. Phys. 6, 1-15. https://doi.org/10.3389/fphy.2018.00049

Shemesh, N., Adiri, T., Cohen, Y., 2011. Probing microscopic architecture of opaque heterogeneous systems using double-pulsed-field-gradient NMR. J. Am. Chem. Soc. 133, 6028-6035. https://doi.org/10.1021/ja200303h 
Shemesh, N., Barazany, D., Sadan, O., Bar, L., Zur, Y., Barhum, Y., Sochen, N., Offen, D., Assaf, Y., Cohen, Y., 2012b. Mapping Apparent Eccentricity and Residual Ensemble Anisotropy in the Gray Matter Using Angular Double-Pulsed-FieldGradient MRI. Magn. Reson. Med. 68, 794-806. https://doi.org/10.1002/mrm.23300

Shemesh, N., Cohen, Y., 2011. Microscopic and compartment shape anisotropies in gray and white matter revealed by angular bipolar double-PFG MR. Magn. Reson. Med. 65, 1216-1227. https://doi.org/10.1002/mrm.22738

Shemesh, N., Jespersen, S.N., Alexander, D.C., Cohen, Y., Drobnjak, I., Dyrby, T.B., Finsterbusch, J., Koch, M.A., Kuder, T., Laun, F., Lawrenz, M., Lundell, H., Mitra, P.P., Nilsson, M., Özarslan, E., Topgaard, D., Westin, C.F., 2016. Conventions and nomenclature for double diffusion encoding NMR and MRI. Magn. Reson. Med. 75, 82-87. https://doi.org/10.1002/mrm.25901

Shemesh, N., Özarslan, E., Adiri, T., Basser, P.J., Cohen, Y., 2010b. Noninvasive bipolar double-pulsed-field-gradient NMR reveals signatures for pore size and shape in polydisperse, randomly oriented, inhomogeneous porous media. J. Chem. Phys. 133. https://doi.org/10.1063/1.3454131

Shemesh, N., Özarslan, E., Basser, P.J., Cohen, Y., 2012a. Accurate noninvasive measurement of cell size and compartment shape anisotropy in yeast cells using double-pulsed field gradient MR. NMR Biomed. 25, 236-246. https://doi.org/10.1002/nbm.1737

Shemesh, N., Özarslan, E., Komlosh, M.E., Basser, P.J., Cohen, Y., 2010a. From singlepulsed field gradient to double-pulsed field gradient MR: Gleaning new microstructural information and developing new forms of contrast in MRI. NMR Biomed. 23, 757-780. https://doi.org/10.1002/nbm.1550

Stejskal, E.O., Tanner, J.E., 1965. Spin Diffusion Measurements: Spin Echoes in the Presence of a Time-Dependent Field Gradient. J. Chem. Phys. 42, 288-292. https://doi.org/10.1192/bjp.111.479.1009-a

Steven, A.J., Zhuo, J., Melhem, E.R., 2014. Diffusion kurtosis imaging: An emerging technique for evaluating the microstructural environment of the brain. Am. J. Roentgenol. 202, 26-33. https://doi.org/10.2214/AJR.13.11365

Struyfs, H., Van Hecke, W., Veraart, J., Sijbers, J., Slaets, S., De Belder, M., Wuyts, L., Peters, B., Sleegers, K., Robberecht, C., Van Broeckhoven, C., De Belder, F., Parizel, P.M., Engelborghs, S., 2015. Diffusion kurtosis imaging: A possible MRI biomarker for AD diagnosis? J. Alzheimer's Dis. 48, 937-948. https://doi.org/10.3233/JAD-150253 
Surova, Y., Nilsson, M., Lampinen, B., Lätt, J., Hall, S., Widner, H., van Westen, D., Hansson, O., 2018. Alteration of putaminal fractional anisotropy in Parkinson's disease: a longitudinal diffusion kurtosis imaging study. Neuroradiology 60, 247254. https://doi.org/10.1007/s00234-017-1971-3

1250

1251

1252

1253

1254

1255

1256

1257

1258

1259

1260

1261

1262

1263

1264

1265

1266

1267

1268

1269

1270

1271

1272

1273

1274

1275

1276

1277

1278

1279

1280

1281

Szczepankiewicz, F., Lasič, S., van Westen, D., Sundgren, P.C., Englund, E., Westin, C.F., Ståhlberg, F., Lätt, J., Topgaard, D., Nilsson, M., 2015. Quantification of microscopic diffusion anisotropy disentangles effects of orientation dispersion from microstructure: Applications in healthy volunteers and in brain tumors. Neuroimage 104, 241-252. https://doi.org/10.1016/j.neuroimage.2014.09.057

Szczepankiewicz, F., Sjölund, J., Ståhlberg, F., Lätt, J., Nilsson, M., 2019a. Tensorvalued diffusion encoding for diffusional variance decomposition (DIVIDE): Technical feasibility in clinical MRI systems. PLoS One 14, 1-20. https://doi.org/10.1371/journal.pone.0214238

Szczepankiewicz, F., van Westen, D., Englund, E., Westin, C.F., Ståhlberg, F., Lätt, J., Sundgren, P.C., Nilsson, M., 2016. The link between diffusion MRI and tumor heterogeneity: Mapping cell eccentricity and density by diffusional variance decomposition (DIVIDE). Neuroimage 142, 522-532. https://doi.org/10.1016/j.neuroimage.2016.07.038

Szczepankiewicz, F., Westin, C., Nilsson, M., 2019b. Maxwell-compensated design of asymmetric gradient waveforms for tensor-valued diffusion encoding. Magn. Reson. Med. Sci. 82, 1424-1437. https://doi.org/10.1002/mrm.27828

Tabesh, A., Jensen, J.H., Ardekani, B.A., Helpern, J.A., 2011. Estimation of tensors and tensor-derived measures in diffusional kurtosis imaging. Magn. Reson. Med. 65, 823-836. https://doi.org/10.1002/mrm.22655

Topgaard, D., 2019. Diffusion tensor distribution imaging. NMR Biomed. 32, 1-12. https://doi.org/10.1002/nbm.4066

Topgaard, D., 2017. Multidimensional diffusion MRI. J. Magn. Reson. 275, 98-113. https://doi.org/10.1016/j.jmr.2016.12.007

Tournier, J.D., Smith, R., Raffelt, D., Tabbara, R., Dhollander, T., Pietsch, M., Christiaens, D., Jeurissen, B., Yeh, C.H., Connelly, A., 2019. MRtrix3: A fast, flexible and open software framework for medical image processing and visualisation. Neuroimage 202, 116137. https://doi.org/10.1016/j.neuroimage.2019.116137

Tustison, N.J., Avants, B.B., Cook, P.A., Zheng, Y., Egan, A., Yushkevich, P.A., Gee, J.C., 2010. N4ITK: Improved N3 bias correction. IEEE Trans. Med. Imaging 29, 1310-1320. https://doi.org/10.1109/TMI.2010.2046908 
Van, A.T., Holdsworth, S.J., Bammer, R., 2014. In Vivo Investigation of Restricted Diffusion in the Human Brain with Optimized Oscillating Diffusion Gradient Encoding. Magn. Reson. Med. 71. https://doi.org/10.1021/nn300902w.Release

van der Kouwe, A.J.W., Benner, T., Salat, D.H., Fischl, B., 2008. Brain morphometry with multiecho MPRAGE. Neuroimage 40, 559-569. https://doi.org/10.1016/j.neuroimage.2007.12.025

Veraart, J., Novikov, D.S., Christiaens, D., Ades-aron, B., Sijbers, J., Fieremans, E., 2016. Denoising of diffusion MRI using random matrix theory. Neuroimage 142, 394-406. https://doi.org/10.1016/j.neuroimage.2016.08.016

Vos, S.B., Tax, C.M.W., Luijten, P.R., Ourselin, S., Leemans, A., Froeling, M., 2017. The importance of correcting for signal drift in diffusion MRI. Magn. Reson. Med. https://doi.org/10.1002/mrm.26124

Wang, J.J., Lin, W.Y., Lu, C.S., Weng, Y.H., Ng, S.H., Wang, C.H., Liu, H.L., Hsieh, R.H., Wan, Y.L., Wai, Y.Y., 2011. Parkinson disease: Diagnostic utility of diffusion kurtosis imaging. Radiology 261, 210-217. https://doi.org/10.1148/radiol.11102277

Westin, C.F., Knutsson, H., Pasternak, O., Szczepankiewicz, F., Özarslan, E., van Westen, D., Mattisson, C., Bogren, M., O’Donnell, L.J., Kubicki, M., Topgaard, D., Nilsson, M., 2016. Q-space trajectory imaging for multidimensional diffusion MRI of the human brain. Neuroimage 135, 345-362. https://doi.org/10.1016/J.NEUROIMAGE.2016.02.039

Westin CF, Szczepankiewicz F, Pasternak O, Ozarslan E, Topgaard D, Knutsson H, Nilsson M. Measurement tensors in diffusion MRI: generalizing the concept of diffusion encoding. Med Image Comput Comput Assist Interv. 2014;17(Pt 3):20916. doi: 10.1007/978-3-319-10443-0_27

Wu, E.X., Cheung, M.M., 2010. MR diffusion kurtosis imaging for neural tissue characterization. NMR Biomed. 23, 836-848. https://doi.org/10.1002/nbm.1506

Yang, G., Tian, Q., Leuze, C., Wintermark, M., McNab, J.A., 2018. Double diffusion encoding MRI for the clinic. Magn. Reson. Med. 80, 507-520. https://doi.org/10.1002/mrm.27043

Yon, M., Bao, Q., Chitrit, O.J., Henriques, R.N., Shemesh, N., Frydman, L., 2020. HighResolution 3D in vivo Brain Diffusion Tensor Imaging at Ultrahigh Fields: Following Maturation on Juvenile and Adult Mice. Front. Neurosci. 14, 1-14. https://doi.org/10.3389/fnins.2020.590900 
brain. Neuroimage 61, 1000-1016.

https://doi.org/10.1016/j.neuroimage.2012.03.072

Zhuo, J., Xu, S., Proctor, J.L., Mullins, R.J., Simon, J.Z., Fiskum, G., Gullapalli, R.P., 2012. Diffusion kurtosis as an in vivo imaging marker for reactive astrogliosis in traumatic brain injury. Neuroimage 59, 467-477. https://doi.org/10.1016/j.neuroimage.2011.07.050 
bioRxiv preprint doi: https://doi.org/10.1101/2021.11.02.466950; this version posted November 4, 2021. The copyright holder for this preprint (which was not certified by peer review) is the author/funder, who has granted bioRxiv a license to display the preprint in perpetuity. It is made available under aCC-BY-NC-ND 4.0 International license. 